\title{
Iron(III)-Catalyzed Chlorination of Activated Arenes
}

\author{
Mohamed A. B. Mostafa, Rosalind M. Bowley, Daugirdas T. Racys, Martyn C. Henry, \\ and Andrew Sutherland*(1)
}

WestCHEM, School of Chemistry, University of Glasgow, The Joseph Black Building, Glasgow G12 8QQ United Kingdom

Supporting Information

ABSTRACT: A general and regioselective method for the chlorination of activated arenes has been developed. The transformation uses iron(III) triflimide as a powerful Lewis acid for the activation of $\mathrm{N}$-chlorosuccinimide and the subsequent chlorination of a wide range of anisole, aniline, acetanilide, and phenol derivatives. The reaction was utilized for the late-stage mono- and dichlorination of a range of target compounds such as the natural product nitrofungin, the antibacterial agent chloroxylenol, and the herbicide chloroxynil. The facile nature of this transformation was demonstrated with the development of one-pot, tandem, iron-catalyzed dihalogenation processes allowing highly regioselective formation of different carbon-halogen bonds. The synthetic utility of the resulting dihalogenated aryl compounds as building blocks was established with the synthesis of natural products and pharmaceutically relevant targets.

\section{INTRODUCTION}

Aromatic chlorides are an important class of compounds, widely used in organic chemistry as synthetic intermediates for coupling or substitution reactions, allowing the preparation of natural products and medicinally active compounds. ${ }^{1}$ Because of the relative stability of the carbon-chlorine bond, aryl chlorides are also found as components in a vast array of natural products (e.g., phenols 1-3, Figure 1$)^{2-5}$ and used as structural

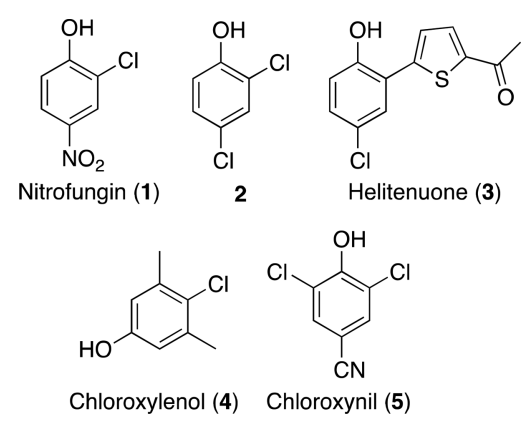

Figure 1. Structures of biologically active chlorobenzenes.

motifs in pharmaceuticals, agrochemicals, and other biologically active compounds. Examples include the fungicide nitrofungin (1), ${ }^{3}$ the broad spectrum antimicrobial agent chloroxylenol (4), ${ }^{6}$ and the pesticide chloroxynil (5).

Due to the prevalence and importance of aryl chlorides, many general methods are known for the synthesis of these compounds. In fact, the halogenation of arenes is a fundamental reaction of organic synthesis. Traditionally, chlorination of aromatic compounds has involved electrophilic aromatic substitution, ${ }^{8}$ the Sandmeyer reaction of anilines, ${ }^{9}$ or a directed ortho-lithiation-chlorination sequence. ${ }^{10}$ Despite the wide- spread use of these methods, they can involve harsh conditions and long reaction times and give poor regioselectivity and control. To overcome these limitations, recent efforts have focused on the development of new chlorination methods, ${ }^{11}$ including transition-metal-catalyzed reactions. ${ }^{12,13}$ In particular, the widespread developments in transition-metal-catalyzed chelation-directed aryl $\mathrm{C}-\mathrm{H}$ activation have led to a number of methods for ortho-chlorination (Scheme 1a). ${ }^{14}$ Recently, a chelation-directed aryl $\mathrm{C}-\mathrm{H}$ activation process for metachlorination was developed by $\mathrm{Yu}$ and co-workers using a palladium-catalyzed pyridone ligand promoted protocol (Scheme 1b). ${ }^{15}$

Scheme 1. Strategies for Regioselective Aryl Chlorination

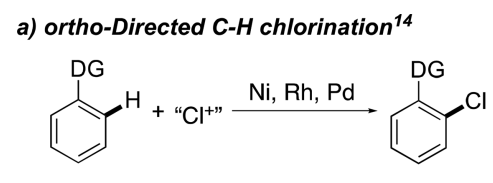

b) Ligand-assisted meta-Directed Chlorination ${ }^{15}$

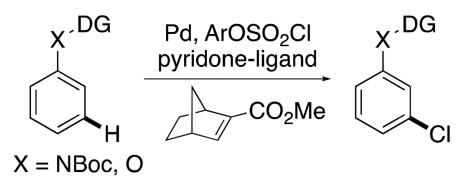

c) This Work: Iron-Catalyzed para-Directed Chlorination

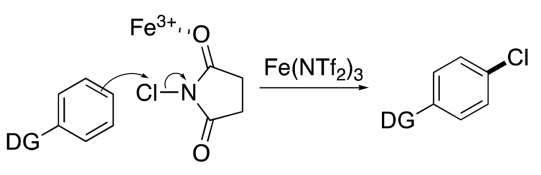

Received: May 18, 2017

Published: June 29, 2017 
We recently reported the use of iron(III) triflimide or silver(I) triflimide as powerful Lewis acids for $\mathrm{N}$-iodosuccinimide activation and the iodination of aryl compounds for application in radionuclide imaging. ${ }^{16}$ This type of transformation was also coupled with a copper-catalyzed Ullmanntype reaction resulting in a one-pot intermolecular aryl $\mathrm{C}-\mathrm{H}$ amination process. ${ }^{17} \mathrm{We}$ were interested in extending this Lewis acid catalyzed activation of $N$-halosuccinimides for the para-chlorination of arenes, resulting in a protocol that would be complementary to the many known ortho-directed chlorination methods. Furthermore, it was proposed that this type of Lewis acid catalyzed chlorination process could be coupled with other halogenation reactions for the regioselective, one-pot dihalogenation of aromatic compounds. We now report the development of a general chlorination protocol for para-substitution of activated arenes (Scheme 1c). In addition to exploring the scope of this process for the preparation of a wide range of synthetic building blocks, natural products, and pharmaceutically important compounds, we also describe the development of a tandem iron(III)-catalyzed chlorinationbromination process for the regioselective dihalogenation of aromatic compounds. The synthetic utility of these compounds for targeted synthesis is also reported.

\section{RESULTS AND DISCUSSION}

The study began with an investigation of the chlorination of anisole. We have previously shown iron(III) triflimide catalyzed bromination and iodination of anisole. ${ }^{16 a, 17}$ During these transformations, iron(III) triflimide was generated in situ using iron(III) chloride (5 mol \%) and the ionic liquid, 1butyl-3-methylimidazolium bis(trifluoromethylsulfonyl)imide ([BMIM] $] \mathrm{NTf}_{2}$ ), which was also used as the reaction solvent. ${ }^{18,19}$ Under the same conditions, it was found that chlorination of anisole (6a) with $N$-chlorosuccinimide (NCS) required more forcing conditions, with a higher temperature of $50{ }^{\circ} \mathrm{C}$ (versus $20{ }^{\circ} \mathrm{C}$ for iodination), to give $100 \%$ conversion after $5 \mathrm{~h}$ (Table 1 , entry 1 ). The presence of iron(III) chloride

Table 1. Optimization of the Iron-Catalyzed Chlorination of Anisole

\begin{tabular}{|c|c|c|c|c|c|c|}
\hline & $6 a$ & $\begin{array}{c}\begin{array}{c}\mathrm{FeCl}_{3}, \mathrm{NCS} \\
{[\mathrm{BMIM}] \mathrm{NTf}_{2}}\end{array} \\
\text { solvent }\end{array}$ & $7 a$ & \begin{tabular}{|c|} 
[BMIN \\
$n-B u-$ \\
$-\mathrm{N}(\mathrm{S}$ \\
\end{tabular} & $\begin{array}{l}\mathrm{NTf}_{2}= \\
-\mathrm{N}_{+}^{+} \\
\mathrm{N}_{-} \\
\left.\mathrm{CF}_{3}\right)_{2}\end{array}$ & \\
\hline entry & $\begin{array}{c}\mathrm{FeCl}_{3} \\
(\mathrm{~mol} \mathrm{\%})\end{array}$ & $\begin{array}{c}{\left[\mathrm{BMIM}^{\mathrm{BMTf}}\right]} \\
\mathrm{NTf}_{2} \\
(\mathrm{~mol} \mathrm{\%})\end{array}$ & solvent & $\begin{array}{l}\text { time } \\
(\mathrm{h})\end{array}$ & $\begin{array}{l}\text { temp } \\
\left({ }^{\circ} \mathrm{C}\right)\end{array}$ & $\begin{array}{c}\text { conv }^{a} \\
(\%)\end{array}$ \\
\hline 1 & 5 & & [BMIM] NTf $_{2}$ & 5 & 50 & 100 \\
\hline 2 & 0 & & [BMIM] $\mathrm{NTf}_{2}$ & 24 & 50 & 0 \\
\hline 3 & 2.5 & 7.5 & $t$-BuOMe & 24 & 50 & 0 \\
\hline 4 & 2.5 & 7.5 & toluene & 24 & 50 & 60 \\
\hline 5 & 2.5 & 7.5 & THF & 24 & 50 & 75 \\
\hline 6 & 2.5 & 7.5 & THF & 18 & 60 & 100 \\
\hline 7 & 5 & 15 & THF & 8 & 60 & 100 \\
\hline
\end{tabular}

${ }^{a}$ Conversions were measured using ${ }^{1} \mathrm{H}$ NMR spectroscopy.

during this transformation is crucial for activation of NCS, as performing the reaction in the absence of $\mathrm{FeCl}_{3}$ under the same conditions $\left(50{ }^{\circ} \mathrm{C},[\mathrm{BMIM}] \mathrm{NTf}_{2}\right.$ as the solvent) showed no reaction after $24 \mathrm{~h}$ (entry 2$).{ }^{20}$ While [BMIM] $\mathrm{NTf}_{2}$ is relatively inexpensive and recyclable and imparts high cohesive pressure to the reaction resulting in fast halogenations, we were interested in developing for the first time a halogenation reaction that was catalytic in both iron(III) chloride and $[\mathrm{BMIM}] \mathrm{NTf}_{2}$. It was proposed that such a process would allow the chlorination of a wider substrate scope and simpler workup and isolation protocols. Therefore, a solvent screen was performed using iron(III) chloride (2.5 mol \%) and [BMIM]$\mathrm{NTf}_{2}$ (7.5 mol \%). Although no conversion was observed using tert-butyl methyl ether (entry 3 ), good reactivity was noted after $24 \mathrm{~h}$ in both toluene and THF (entries 4 and 5). Using THF as the optimal solvent, an increase in temperature to 60 ${ }^{\circ} \mathrm{C}$ gave complete conversion, combined with a shorter reaction time of $18 \mathrm{~h}$ (entry 6). It was also noted that a doubling of catalyst loading of both iron(III) trichloride and [BMIM] NTf at $60{ }^{\circ} \mathrm{C}$ gave a shorter reaction time of $8 \mathrm{~h}$ (entry 7 ). While the conditions with low catalyst loadings (entry 6) were deemed suitable for most activated aryl compounds, it was felt necessary to demonstrate the viability of procedures with higher catalyst loadings (entries 1 and 7) that would likely be required for more challenging substrates.

The optimized procedure (Table 1, entry 6) was then used to explore the chlorination of activated arenes (Scheme 2). In general, the transformation was found to be compatible with a wide range of functional groups and could be used for the efficient monochlorination of anisole, phenol, aniline, and acetanilide compounds $(7 \mathbf{a}-\mathbf{q})$. In contrast to iodination using

Scheme 2. Scope of Iron(III) Triflimide Catalyzed Chlorination $^{a}$
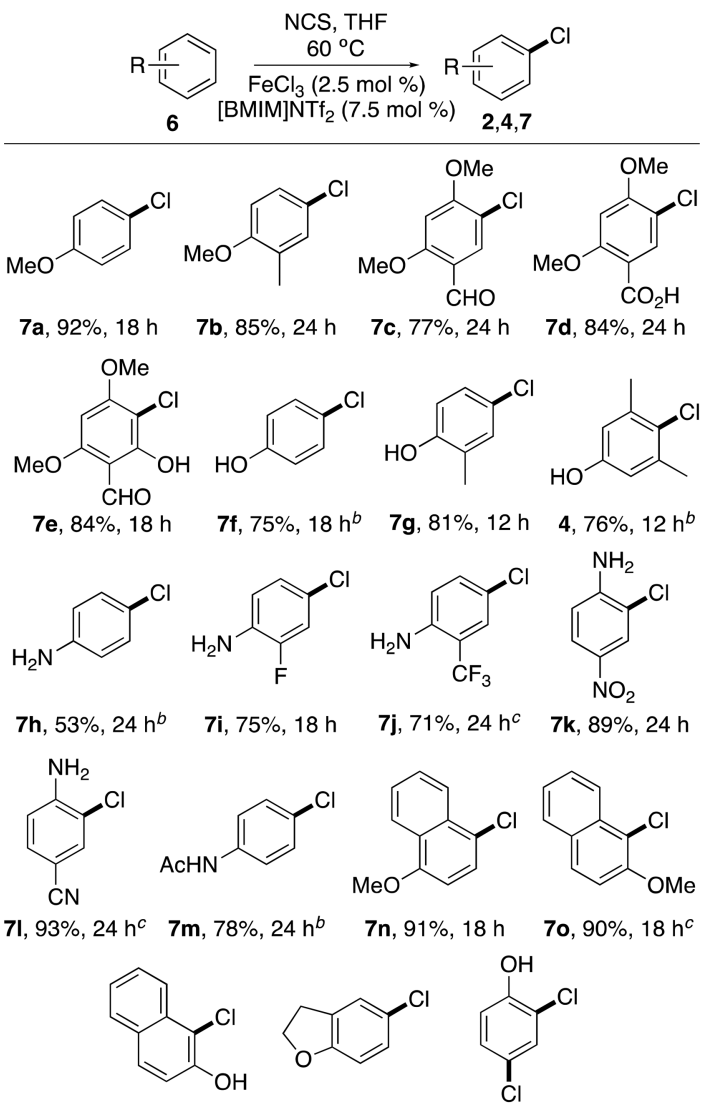

7 p, $97 \%, 18$ h $\quad 7 q, 86 \%, 18$ h $\quad 2,81 \%, 48$ h

${ }^{a}$ Isolated yields are shown. ${ }^{b}$ Ortho-chlorinated products were observed (20\% for $\mathbf{6 f}$, $\mathbf{m}$-xylenol, and $\mathbf{6 m}$; $30 \%$ for $\mathbf{6 h}$ ). ${ }^{c}$ Reaction was performed at $70{ }^{\circ} \mathrm{C}$ using iron(III) trichloride $(5 \mathrm{~mol} \%)$ and $[\mathrm{BMIM}] \mathrm{NTf}_{2}(15 \mathrm{~mol} \%)$. 
iron(III) triflimide where only para products were formed, ${ }^{16 a}$ chlorination of several monosubstituted substrates [e.g., phenol (6f), aniline (6h), and acetanilide $(6 \mathbf{m})]$ yielded the orthoisomer as a byproduct. This is likely due to a combination of the relatively small size of the chlorinating agent and the more forcing conditions required for this transformation. Nevertheless, the major para-isomers were easily separated and isolated in good yields (53-78\%). For di-, tri-, and tetrasubstituted substrates, only the para-chlorinated products were formed, as observed by NMR spectroscopy of the crude reaction mixture. Similarly, for compounds with the parapositioned blocked, ortho-chlorinated compounds were cleanly formed in high yield and as single regioisomers. While the optimized procedure $\left(2.5 \mathrm{~mol} \%\right.$ of $\mathrm{FeCl}_{3}$ ) was effective for most substrates, including $\mathbf{6 k}$ bearing the deactivating paranitro group, several other aryl compounds containing strongly electron-withdrawing groups (e.g., $\mathbf{6 j}$ and $\mathbf{6 1}$ ) required higher catalyst loading of both $\mathrm{FeCl}_{3}(5 \mathrm{~mol} \%)$ and [BMIM] $\mathrm{NTf}_{2}(15$ mol \%) and a higher reaction temperature $\left(70{ }^{\circ} \mathrm{C}\right)$ for efficient chlorination. Other arenes, such as naphthalenes $\mathbf{6 n}-\mathbf{p}$ and 2,3dihydrobenzofuran (6q), were also chlorinated, yielding single regioisomers in high yields. In addition to using this method for the preparation of antiseptic agent chloroxylenol (4), ${ }^{6}$ reaction of phenol (6f) with 2 equiv of NCS allowed efficient dichlorination and the synthesis of 2,4-dichlorophenol (2), a compound isolated from soil Penicillium, that is used as a growth hormone and as an intermediate for the preparation of herbicides. ${ }^{4}$

A number of aryl compounds with deactivating groups gave poor or modest conversion when using the procedures involving catalytic $[\mathrm{BMIM}] \mathrm{NTf}_{2}$. Therefore, an alternative procedure was utilized for the chlorination of these compounds. It was found that using $\mathrm{FeCl}_{3}(5 \mathrm{~mol} \%)$ and $[\mathrm{BMIM}] \mathrm{NTf}_{2}$ as the solvent, at $70{ }^{\circ} \mathrm{C}$, gave complete conversion to the monochlorinated products $(\mathbf{1}, \mathbf{5}$, and $\mathbf{7} \mathbf{r}-\mathbf{u}$, Scheme 3$)$. While

Scheme 3. Iron(III) Triflimide Catalyzed Chlorination in $[\mathrm{BMIM}] \mathrm{NTf}_{2}{ }^{a}$

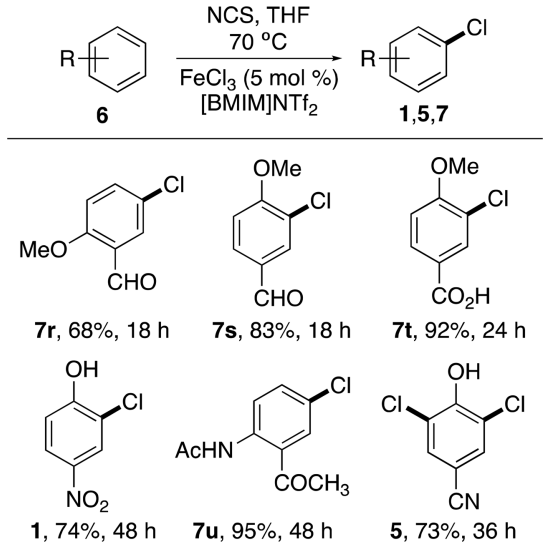

${ }^{a}$ Isolated yields are shown.

the reactions required relatively long reaction times, the products were cleanly isolated in high yields. This allowed the efficient synthesis of 3-chloro-4-methoxybenzaldehyde $(7 \mathbf{s}),^{21}$ a major metabolite from soil Lepista nuda, as well as nitrofungin (1), a compound isolated from Stephanospora caroticolor, which was previously used as a fungicide. ${ }^{3}$ These conditions were also effective for the dichlorination of 4- hydroxybenzonitrile, producing the pesticide chloroxynil (5) in $73 \%$ yield.

The examination of the scope of this protocol clearly demonstrated that iron(III) triflimide catalyzed chlorination, compared to bromination or iodination, required higher temperatures and longer reaction times. This was expected due to the stronger $\mathrm{N}-\mathrm{Cl}$ bond of NCS. It was decided to take advantage of the reactivity difference and the clean production of halogenated products to use this protocol for the design of one-pot multistep halogenation processes. It was proposed that a single loading of iron(III) triflimide could be used to catalyze multiple halogenation reactions, allowing the selective preparation of carbon-halogen bonds with high regiocontrol. It was believed that the products of such a process could be exploited by using the differences in reactivity of the carbon-halogen bonds to introduce further functionality. As a proof of concept, anisole (6a) was subjected to a bromination reaction followed by chlorination (Scheme 4). Using iron(III) chloride (5 mol \%)

Scheme 4. Tandem Iron(III)-Catalyzed Regioselective Dihalogenation $^{a}$

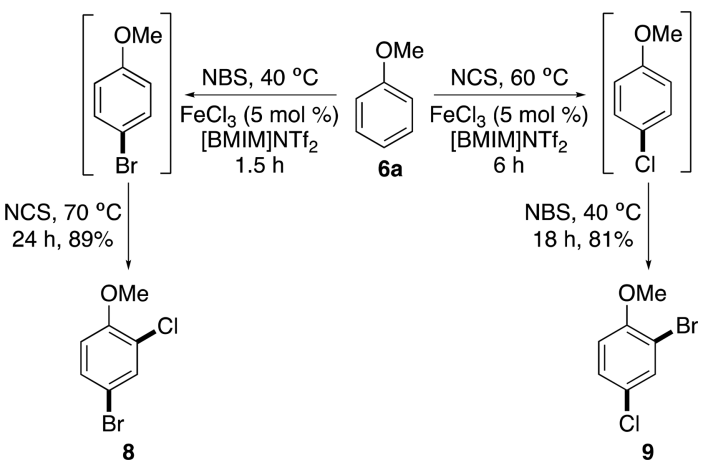

${ }^{a}$ Isolated yields are shown

in $[\mathrm{BMIM}] \mathrm{NTf}_{2}$ as the solvent at $40{ }^{\circ} \mathrm{C}$ gave the parabrominated product after $1.5 \mathrm{~h}$. Addition of NCS to the reaction mixture and an increase in temperature to $70{ }^{\circ} \mathrm{C}$ allowed complete ortho-chlorination after $24 \mathrm{~h}$ and the isolation of 8 in $89 \%$ yield. The order of halogenation could also be reversed. Chlorination at higher temperature $\left(60^{\circ} \mathrm{C}\right)$ followed by bromination at $40{ }^{\circ} \mathrm{C}$ gave 2-bromo-4-chloroanisole (9) in $81 \%$ yield. The highly regioselective, clean generation of both the intermediate and the final product allowed the scale up of this process and the multigram synthesis of 9 .

Having used the tandem iron(III)-catalyzed halogenation process for the one-pot synthesis of 9, we wanted to demonstrate the synthetic utility of such compounds, in particular, for the selective functionalization of the $\mathrm{C}-\mathrm{Br}$ bond and the preparation of biologically relevant chlorobenzene targets. 2-Bromo-4-chloroanisole (9) was found to be a good substrate for copper(I)-catalyzed $\mathrm{N}$-arylation reactions (Scheme 5). Using 4-chlorobenzenesulfonamide as the nucleophile gave 10, a compound with antibacterial activity, in $65 \%$ yield. $^{22}$ A similar copper(I)-catalyzed $N$-arylation reaction with benzenesulfonamide followed by $N$-methylation gave cholinesterase inhibitor $\mathbf{1 2}$ in good overall yield. ${ }^{23} 2$ Bromo-4-chloroanisole (9) was also used for a novel synthesis of helitenuone (3), a thiophene-derived chlorophenol isolated from Helichrysum species. ${ }^{5}$ The only other synthesis of this natural product involved a six-step sequence from a chlorinated benzaldehyde derivative, which gave helitenuone (3) in $7 \%$ 
Scheme 5. Synthetic Applications of 2-Bromo-4-chloroanisole (9) ${ }^{a}$

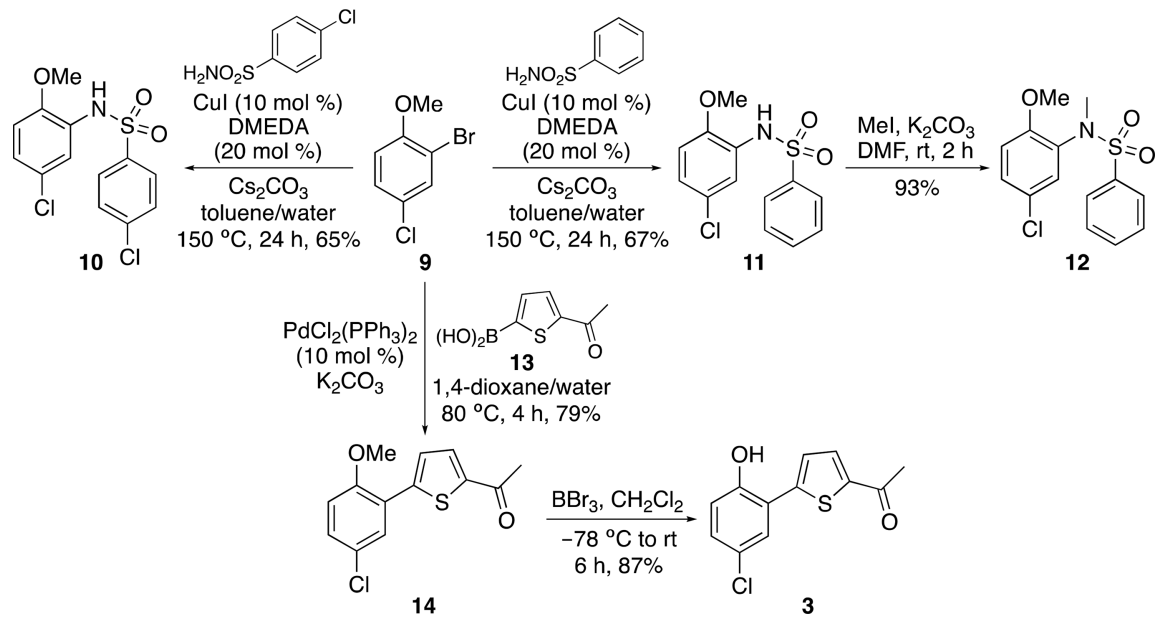

${ }^{a}$ Isolated yields are shown.

overall yield. $^{5 \mathrm{~b}}$ In this current study, 2-bromo-4-chloroanisole (9) was subjected to a Suzuki-Miyaura reaction with commercially available thiophene-derived boronic acid 13, under standard conditions. This gave coupled product 14 in $79 \%$ yield. Deprotection of the phenol moiety using boron tribromide completed the three-pot synthesis of helitenuone (3) in $56 \%$ overall yield from anisole (6a).

\section{CONCLUSIONS}

In summary, methods for the chlorination of arenes using iron(III) triflimide activation of NCS have been developed. In particular, a procedure that utilizes catalytic amounts of both iron(III) chloride and [BMIM] $\mathrm{NTf}_{2}$ was optimized for the regioselective monochlorination of activated arene substrates, while the use of higher loadings and the ionic liquid as a solvent was effective for more deactivated aromatic compounds. The reactions were also extended for dihalogenation, including the development of a selective one-pot, tandem, iron(III)-catalyzed process where different halogens could be introduced in a highly regioselective manner. The synthetic utility of both the single-step chlorination and the one-pot dihalogenations has been demonstrated with the rapid and efficient synthesis of a range of chlorobenzene-containing natural products and biologically active targets. In particular, the three-pot regioselective synthesis of helitenuone (3) demonstrates how highly functional aromatic compounds can be quickly accessed using these methods. Further studies to extend these strategies and concepts for the synthesis of other halogenated arenes are currently underway.

\section{EXPERIMENTAL SECTION}

All reagents and starting materials were obtained from commercial sources and used as received. The iron(III) chloride used in this study is reagent grade (97\%). All dry solvents were purified using a solvent purification system. All reactions were performed under an atmosphere of argon unless otherwise mentioned. Brine refers to a saturated solution of sodium chloride. Flash column chromatography was performed using silica gel $60(35-70 \mu \mathrm{m})$. Aluminum-backed plates precoated with silica gel $60 \mathrm{~F}_{254}$ were used for thin-layer chromatography and were visualized with a UV lamp or by staining with potassium permanganate. ${ }^{1} \mathrm{H}$ NMR spectra were recorded on a NMR spectrometer at either 400 or $500 \mathrm{MHz}$, and data are reported as follows: chemical shift in $\mathrm{ppm}$ relative to tetramethylsilane as the internal standard, multiplicity $(\mathrm{s}=$ singlet, $\mathrm{d}=$ doublet, $\mathrm{t}=$ triplet, $\mathrm{q}=$ quartet, $\mathrm{m}=$ multiplet or overlap of nonequivalent resonances, integration). ${ }^{13} \mathrm{C}$ NMR spectra were recorded on a NMR spectrometer at either 101 or $126 \mathrm{MHz}$, and data are reported as follows: chemical shift in ppm relative to tetramethylsilane or the solvent $\left(\mathrm{CDCl}_{3}, \delta 77.0\right.$ ppm or DMSO- $d_{6}, \delta 39.5 \mathrm{ppm}$ ) as internal standard, multiplicity with respect to hydrogen (deduced from DEPT experiments, $\mathrm{C}, \mathrm{CH}, \mathrm{CH}_{2}$, or $\mathrm{CH}_{3}$ ). Conversions (Table 1) were measured by ${ }^{1} \mathrm{H}$ NMR spectroscopy using dimethyl sulfone as an internal standard. Infrared spectra were recorded on a FTIR spectrometer; wavenumbers are indicated in $\mathrm{cm}^{-1}$. Mass spectra were recorded using electron impact, chemical ionization, or electrospray techniques. HRMS spectra were recorded using a dual-focusing magnetic analyzer mass spectrometer. Melting points are uncorrected.

General Chlorination Procedure A. Iron(III) chloride (0.0250 $\mathrm{mmol}$ ) was dissolved in 1-butyl-3-methylimidazolium bis(trifluoromethanesulfonyl)imide $(0.0750 \mathrm{mmol})$, stirred for $0.5 \mathrm{~h}$ at room temperature, and then added to a solution of $\mathrm{N}$-chlorosuccinimide $(1.05 \mathrm{mmol})$ in tetrahydrofuran $(0.6 \mathrm{~mL})$ under an atmosphere of air. The substrate $(1.00 \mathrm{mmol})$ was then added, and the reaction mixture was heated to $60{ }^{\circ} \mathrm{C}$, resulting in a homogeneous solution. Upon completion, the reaction mixture was cooled to room temperature, diluted with ethyl acetate $(10 \mathrm{~mL})$, and washed with a $1 \mathrm{M}$ sodium thiosulfate solution $(10 \mathrm{~mL})$ and brine $(10 \mathrm{~mL})$. The organic phase was dried $\left(\mathrm{MgSO}_{4}\right)$, filtered, and concentrated in vacuo. The crude product was purified by flash column chromatography.

General Chlorination Procedure B. N-Chlorosuccinimide (1.05 $\mathrm{mmol})$ was added to a solution of iron(III) chloride $(0.0500 \mathrm{mmol})$ in 1-butyl-3-methylimidazolium bis(trifluoromethanesulfonyl)imide (0.3 $\mathrm{mL}$ ) under an atmosphere of air. The mixture was stirred at room temperature for $0.5 \mathrm{~h}$ before the substrate $(1.00 \mathrm{mmol})$ in acetonitrile $(0.1 \mathrm{~mL})$ was added. The reaction mixture was heated to $70{ }^{\circ} \mathrm{C}$, resulting in a homogeneous mixture. Upon completion, the reaction mixture was then extracted with $5 \%$ ethyl acetate in hexane $(3 \times 50$ $\mathrm{mL}$ ) using sonication in a water bath for $0.1 \mathrm{~h}$. The suspension was washed with an aqueous solution of $1 \mathrm{M}$ sodium thiosulfate $(10 \mathrm{~mL})$ and brine $(10 \mathrm{~mL})$, dried $\left(\mathrm{MgSO}_{4}\right)$, and then filtered through a pad of Celite. The solvent was removed under reduced pressure, and the crude product was purified by flash column chromatography.

Workup for Carboxylic Acids and Phenol Derivatives. The reaction mixture was diluted with dichloromethane $(10 \mathrm{~mL})$ and extracted with an aqueous solution of $1 \mathrm{M}$ sodium hydroxide $(20 \mathrm{~mL})$. The aqueous phase was separated, acidified with $1 \mathrm{M}$ hydrochloric acid, and extracted with dichloromethane $(2 \times 50 \mathrm{~mL})$. The organic phase was dried over $\mathrm{MgSO}_{4}$ and filtered, and the solvent was removed under reduced pressure. The crude product was purified by flash column chromatography. 
4-Chloroanisole $(7 a) .^{24}$ The reaction was performed as described in general procedure $\mathrm{A}$ using anisole $(6 \mathrm{a})(108 \mu \mathrm{L}, 1.00 \mathrm{mmol})$. The reaction mixture was heated to $60{ }^{\circ} \mathrm{C}$ for $18 \mathrm{~h}$. Purification by flash column chromatography (petroleum ether/ethyl acetate 19:1) gave 4chloroanisole (7a) (131 mg, 92\%) as a colorless oil. Spectroscopic data were consistent with the literature: ${ }^{24}{ }^{1} \mathrm{H} \mathrm{NMR}\left(500 \mathrm{MHz}, \mathrm{CDCl}_{3}\right) \delta$ $3.77(\mathrm{~s}, 3 \mathrm{H}), 6.80-6.85(\mathrm{~m}, 2 \mathrm{H}), 7.21-7.26(\mathrm{~m}, 2 \mathrm{H}) ;{ }^{13} \mathrm{C}$ NMR $(126$ $\left.\mathrm{MHz}, \mathrm{CDCl}_{3}\right) \delta 55.5\left(\mathrm{CH}_{3}\right), 115.2(2 \times \mathrm{CH}), 125.5(\mathrm{C}), 129.3(2 \times$ $\mathrm{CH}), 158.2$ (C); MS (EI) $m / z 142\left(\mathrm{M}^{+}, 100\right), 127$ (46), 99 (39), 84 (16).

4-Chloro-2-methylanisole (7b). ${ }^{25}$ The reaction was performed as described in general procedure A using 2-methylanisole (6b) $(124 \mu \mathrm{L}$, $1.00 \mathrm{mmol}$ ). The reaction mixture was heated to $60{ }^{\circ} \mathrm{C}$ for $24 \mathrm{~h}$. Purification by flash column chromatography (petroleum ether/ethyl acetate, 4:1) gave 4-chloro-2-methylanisole (7b) $(133 \mathrm{mg}, 85 \%)$ as a yellow oil. Spectroscopic data were consistent with the literature: ${ }^{25}{ }^{1} \mathrm{H}$ $\operatorname{NMR}\left(400 \mathrm{MHz}, \mathrm{CDCl}_{3}\right) \delta 2.21(\mathrm{~s}, 3 \mathrm{H}), 3.81(\mathrm{~s}, 3 \mathrm{H}), 6.73(\mathrm{~d}, J=9.2$ $\mathrm{Hz}, 1 \mathrm{H}), 7.09-7.15(\mathrm{~m}, 2 \mathrm{H}) ;{ }^{13} \mathrm{C}$ NMR $\left(101 \mathrm{MHz}, \mathrm{CDCl}_{3}\right) \delta 16.2$ $\left(\mathrm{CH}_{3}\right), 55.6\left(\mathrm{CH}_{3}\right), 111.1(\mathrm{CH}), 125.1(\mathrm{C}), 126.4(\mathrm{CH}), 128.6(\mathrm{C})$, $130.5(\mathrm{CH}), 156.5(\mathrm{C}) ; \mathrm{MS}(\mathrm{EI}) \mathrm{m} / z 156\left(\mathrm{M}^{+}, 100\right), 141(63), 83$ (35), 77 (45), 57 (38).

5-Chloro-2,4-dimethoxybenzaldehyde (7c). ${ }^{26}$ The reaction was performed as described in general procedure A using 2,4dimethoxybenzaldehyde $(6 \mathrm{c})(166 \mathrm{mg}, 1.00 \mathrm{mmol})$. The reaction mixture was heated to $60{ }^{\circ} \mathrm{C}$ for $24 \mathrm{~h}$. Purification by flash column chromatography (petroleum ether/ethyl acetate 10:1) gave 5-chloro2,4-dimethoxybenzaldehyde (7c) (154 mg, 77\%) as a white solid. Spectroscopic data were consistent with the literature: ${ }^{26} \mathrm{mp} 130-132$ ${ }^{\circ} \mathrm{C}$; ${ }^{1} \mathrm{H}$ NMR (500 MHz, CDCl $) \delta 3.93(\mathrm{~s}, 3 \mathrm{H}), 3.97$ (s, 3H), $6.46(\mathrm{~s}$, $1 \mathrm{H}), 7.81(\mathrm{~s}, 1 \mathrm{H}), 10.23(\mathrm{~s}, 1 \mathrm{H}) ;{ }^{13} \mathrm{C} \mathrm{NMR}\left(126 \mathrm{MHz}, \mathrm{CDCl}_{3}\right) \delta 56.0$ $\left(\mathrm{CH}_{3}\right), 56.4\left(\mathrm{CH}_{3}\right), 95.7(\mathrm{CH}), 115.3(\mathrm{C}), 118.7(\mathrm{C}), 129.7(\mathrm{CH})$, 160.8 (C), $162.4(\mathrm{C}), 187.2(\mathrm{CH})$; MS (ESI) $m / z 223\left(\mathrm{MNa}^{+}, 100\right)$.

5-Chloro-2,4-dimethoxybenzoic Acid (7d). The reaction was performed as described in general procedure A using 2,4dimethoxybenzoic acid (6d) $(182 \mathrm{mg}, 1.00 \mathrm{mmol})$. The reaction mixture was heated to $60{ }^{\circ} \mathrm{C}$ for $24 \mathrm{~h}$. Purification by flash column chromatography (dichloromethane/methanol, 19:1) gave 5-chloro2,4-dimethoxybenzoic acid (7d) $(181 \mathrm{mg}, 84 \%)$ as a white solid: $\mathrm{mp}$ 166-168 ${ }^{\circ} \mathrm{C}$; IR (neat) 2970, 2569, 1694, 1599, 1243, 1213, 1021, 904, 820, $727 \mathrm{~cm}^{-1}$; ${ }^{1} \mathrm{H}$ NMR (500 MHz, $\left.\mathrm{CDCl}_{3}\right) \delta 3.98(\mathrm{~s}, 3 \mathrm{H}), 4.08$ $(\mathrm{s}, 3 \mathrm{H}), 6.53(\mathrm{~s}, 1 \mathrm{H}), 8.13(\mathrm{~s}, 1 \mathrm{H}) ;{ }^{13} \mathrm{C} \mathrm{NMR}\left(126 \mathrm{MHz}^{1} \mathrm{CDCl}_{3}\right) \delta$ $56.6\left(\mathrm{CH}_{3}\right), 57.1\left(\mathrm{CH}_{3}\right), 96.0(\mathrm{CH}), 110.6(\mathrm{C}), 116.1(\mathrm{C}), 134.5$ $(\mathrm{CH}), 158.4$ (C), 160.0 (C), $164.6(\mathrm{C})$; MS (EI) $\mathrm{m} / z 218\left(\mathrm{M}^{+}, 36\right)$, 216 (100), 199 (47), 169 (42), 142 (22), 78 (27), 63 (32); HRMS (EI) calcd for $\mathrm{C}_{9} \mathrm{H}_{9}{ }^{37} \mathrm{ClO}_{4}\left(\mathrm{M}^{+}\right)$218.0163, found 218.0167.

5-Chloro-2,4-dimethoxy-6-hydroxybenzaldehyde (7e). The reaction was performed as described in general procedure A using 2,4dimethoxy-6-hydroxybenzaldehyde (6e) $(182 \mathrm{mg}, 1.00 \mathrm{mmol})$. The reaction mixture was heated to $60{ }^{\circ} \mathrm{C}$ for $18 \mathrm{~h}$. Purification by flash column chromatography (petroleum ether/ethyl acetate, 7:3) gave 5chloro-2,4-dimethoxy-6-hydroxybenzaldehyde (7e) (182 $\mathrm{mg}, 84 \%)$ as a light yellow solid: $\mathrm{mp} 184-186{ }^{\circ} \mathrm{C}$; IR (neat) 2953, 1631, 1595, 1470, 1453, 1417, 1410, 1292, 1227, 1186, 1119, 1100, 790, $731 \mathrm{~cm}^{-1}$; ${ }^{1} \mathrm{H}$ NMR (500 MHz, $\left.\mathrm{CDCl}_{3}\right) \delta 3.91(\mathrm{~s}, 3 \mathrm{H}), 3.97$ (s, 3H), $5.99(\mathrm{~s}$, $1 \mathrm{H}), 10.09(\mathrm{~s}, 1 \mathrm{H}), 12.80(\mathrm{~s}, 1 \mathrm{H}) ;{ }^{13} \mathrm{C} \mathrm{NMR}\left(126 \mathrm{MHz}, \mathrm{CDCl}_{3}\right) \delta$ $56.0\left(\mathrm{CH}_{3}\right), 56.5\left(\mathrm{CH}_{3}\right), 86.7(\mathrm{CH}), 101.4(\mathrm{C}), 106.1(\mathrm{C}), 160.3(\mathrm{C})$, 162.5 (C), 162.8 (C), $192.0(\mathrm{CH})$; MS (ESI) $m / z 239\left(\mathrm{MNa}^{+}, 100\right)$; HRMS (ESI) calcd for $\mathrm{C}_{9} \mathrm{H}_{9}{ }^{35} \mathrm{ClNaO}_{4}\left(\mathrm{MNa}^{+}\right)$239.0082, found 239.0083.

4-Chlorophenol (7f). ${ }^{27}$ The reaction was performed as described in general procedure A using phenol $(6 \mathrm{f})(94 \mathrm{mg}, 1.0 \mathrm{mmol})$. The reaction mixture was heated to $60{ }^{\circ} \mathrm{C}$ for $18 \mathrm{~h}$. Purification by flash column chromatography (petroleum ether/ethyl acetate, 19:1) gave 4chlorophenol (7f) (96 mg, 75\%) as a colorless oil. Spectroscopic data were consistent with the literature: ${ }^{27}{ }^{1} \mathrm{H}$ NMR $\left(500 \mathrm{MHz}, \mathrm{CDCl}_{3}\right) \delta$ 4.77 (br s, $1 \mathrm{H}), 6.74-6.79(\mathrm{~m}, 2 \mathrm{H}), 7.17-7.22(\mathrm{~m}, 2 \mathrm{H}) ;{ }^{13} \mathrm{C} \mathrm{NMR}$ $\left(126 \mathrm{MHz}, \mathrm{CDCl}_{3}\right) \delta 116.7(2 \times \mathrm{CH}), 125.8(\mathrm{C}), 129.5(2 \times \mathrm{CH})$, 154.0 (C); MS (EI) $m / z 128\left(\mathrm{M}^{+}, 100\right), 102$ (34), 84 (90), 66 (98), $57(27)$.
4-Chloro-2-methylphenol (7g). ${ }^{28}$ The reaction was performed as described in general procedure A using 2-methylphenol (6g) (108 mg, $1.00 \mathrm{mmol})$. The reaction mixture was heated to $60{ }^{\circ} \mathrm{C}$ for $12 \mathrm{~h}$. Purification by flash column chromatography (petroleum ether/ethyl acetate, 19:1) gave 4-chloro-2-methylphenol $(7 \mathrm{~g})(115 \mathrm{mg}, 81 \%)$ as a yellow solid: $\mathrm{mp} 46-48{ }^{\circ} \mathrm{C}$ (lit. ${ }^{28} \mathrm{mp} 46-47{ }^{\circ} \mathrm{C}$ ); ${ }^{1} \mathrm{H}$ NMR (500 $\left.\mathrm{MHz}, \mathrm{CDCl}_{3}\right) \delta 2.22(\mathrm{~s}, 3 \mathrm{H}), 5.02(\mathrm{br} \mathrm{s}, 1 \mathrm{H}), 6.69(\mathrm{~d}, J=8.5 \mathrm{~Hz}, 1 \mathrm{H})$, $7.03(\mathrm{dd}, J=8.5,2.7 \mathrm{~Hz}, 1 \mathrm{H}), 7.10(\mathrm{~d}, J=2.7 \mathrm{~Hz}, 1 \mathrm{H}) ;{ }^{13} \mathrm{C} \mathrm{NMR}$ $\left(126 \mathrm{MHz}, \mathrm{CDCl}_{3}\right) \delta 15.7\left(\mathrm{CH}_{3}\right), 116.1(\mathrm{CH}), 125.3(\mathrm{C}), 125.8(\mathrm{C})$, $126.8(\mathrm{CH}), 130.7(\mathrm{CH}), 152.3(\mathrm{C})$; MS (CI) $\mathrm{m} / z 143\left(\mathrm{MH}^{+}, 48\right)$, 113 (45), 97 (40), 85 (68), 71 (100), 69 (54).

4-Chloro-3,5-dimethylphenol (4). ${ }^{29}$ The reaction was performed as described in general procedure A using 3,5-dimethylphenol $(122 \mathrm{mg}$, $1.00 \mathrm{mmol})$. The reaction mixture was heated to $60{ }^{\circ} \mathrm{C}$ for $12 \mathrm{~h}$. Purification by flash column chromatography (petroleum ether/ethyl acetate, 19:1) gave 4-chloro-3,5-dimethylphenol (4) (119 mg, 76\%) as a white solid: $\mathrm{mp} 112-114{ }^{\circ} \mathrm{C}$ (lit. $\left.{ }^{29} \mathrm{mp} 116{ }^{\circ} \mathrm{C}\right) ;{ }^{1} \mathrm{H}$ NMR (500 $\left.\mathrm{MHz}_{\mathrm{CDCl}}\right) \delta 2.32(\mathrm{~s}, 6 \mathrm{H}), 4.72(\mathrm{~s}, 1 \mathrm{H}), 6.58(\mathrm{~s}, 2 \mathrm{H}) ;{ }^{13} \mathrm{C} \mathrm{NMR}$ $\left(126 \mathrm{MHz}, \mathrm{CDCl}_{3}\right) \delta 20.8\left(2 \times \mathrm{CH}_{3}\right), 115.3(2 \times \mathrm{CH}), 126.3(\mathrm{C})$, $137.4(2 \times \mathrm{C}), 153.2(\mathrm{C})$; MS (CI) m/z $157\left(\mathrm{MH}^{+}, 26\right), 113(66), 97$ (43), 85 (69), 71 (100), 69 (53).

4-Chloroaniline ( $7 h) .{ }^{30}$ The reaction was performed as described in general procedure A using aniline $(6 \mathbf{h})(91 \mu \mathrm{L}, 1.0 \mathrm{mmol})$. The reaction mixture was heated to $40{ }^{\circ} \mathrm{C}$ for $24 \mathrm{~h}$. Purification by flash column chromatography (petroleum ether/ethyl acetate, 19:1) gave 4chloroaniline $(7 \mathrm{~h})(67 \mathrm{mg}, 53 \%)$ as a yellow solid: $\mathrm{mp} 58-60{ }^{\circ} \mathrm{C}$ (lit. $\left.{ }^{30} \mathrm{mp} 63-65{ }^{\circ} \mathrm{C}\right) ;{ }^{1} \mathrm{H}$ NMR $\left(400 \mathrm{MHz}, \mathrm{CDCl}_{3}\right) \delta 3.64$ (br s, $\left.2 \mathrm{H}\right)$, 6.56-6.65 (m, 2H), 7.07-7.14 (m, 2H); ${ }^{13} \mathrm{C}$ NMR (101 MHz, $\left.\mathrm{CDCl}_{3}\right) \delta 116.3(2 \times \mathrm{CH}), 123.3(\mathrm{C}), 129.2(2 \times \mathrm{CH}), 145.1(\mathrm{C})$; MS (EI) $m / z 127$ ( $\left.\mathrm{M}^{+}, 100\right), 92$ (16), 84 (19), 64 (19).

4-Chloro-2-fluoroaniline (7i). ${ }^{31}$ The reaction was performed as described in general procedure A using 2-fluoroaniline $(6 \mathbf{6})(111 \mathrm{mg}$, $1.00 \mathrm{mmol}$ ). The reaction mixture was heated to $60{ }^{\circ} \mathrm{C}$ for $18 \mathrm{~h}$. Purification by flash column chromatography (petroleum ether/ethyl acetate, 19:1) gave 4-chloro-2-fluoroaniline (7i) $(109 \mathrm{mg}, 75 \%)$ as a brown oil. Spectroscopic data were consistent with the literature: ${ }^{31}{ }^{1} \mathrm{H}$

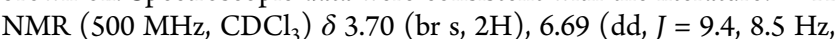
$1 \mathrm{H}), 6.92(\mathrm{ddd}, J=8.5,2.3,1.2 \mathrm{~Hz}, 1 \mathrm{H}), 7.00(\mathrm{dd}, J=10.8,2.3 \mathrm{~Hz}$, $1 \mathrm{H}) ;{ }^{13} \mathrm{C}$ NMR $\left(126 \mathrm{MHz} \mathrm{CDCl}_{3}\right) \delta 115.9\left(\mathrm{CH}, \mathrm{d},{ }^{2} J_{\mathrm{CF}}=22.0 \mathrm{~Hz}\right)$, $117.3\left(\mathrm{CH}, \mathrm{d},{ }^{3} J_{\mathrm{CF}}=4.4 \mathrm{~Hz}\right), 122.5\left(\mathrm{C}, \mathrm{d},{ }^{3} J_{\mathrm{CF}}=9.2 \mathrm{~Hz}\right), 124.5(\mathrm{CH}$, d, $\left.{ }^{4} J_{\mathrm{CF}}=3.6 \mathrm{~Hz}\right), 133.3\left(\mathrm{C}, \mathrm{d},{ }^{2} J_{\mathrm{CF}}=13.0 \mathrm{~Hz}\right), 151.2\left(\mathrm{C}, \mathrm{d},{ }^{1} J_{\mathrm{CF}}=\right.$ $242.3 \mathrm{~Hz}$ ); MS (EI) $\mathrm{m} / z 145\left(\mathrm{M}^{+}, 32\right), 84(100)$.

4-Chloro-2-trifluoromethylaniline (7j). ${ }^{32}$ The reaction was performed as described in general procedure A using 2-(trifluoromethyl)aniline (6j) $(126 \mu \mathrm{L}, 1.00 \mathrm{mmol})$, iron(III) chloride $(8.00 \mathrm{mg}, 0.0500$ $\mathrm{mmol})$, and [BMIM] NTf $\mathrm{N}_{2}(44.0 \mu \mathrm{L}, 0.150 \mathrm{mmol})$ in tetrahydrofuran $(600 \mu \mathrm{L})$. The reaction mixture was heated to $70{ }^{\circ} \mathrm{C}$ for $24 \mathrm{~h}$. Purification by flash column chromatography (petroleum ether/ethyl acetate, 7:3) gave 4-chloro-2-(trifluoromethyl)aniline (7j) (139 mg, $71 \%)$ as a pale yellow oil. Spectroscopic data were consistent with the literature: ${ }^{32}{ }^{1} \mathrm{H}$ NMR $\left(500 \mathrm{MHz}, \mathrm{CDCl}_{3}\right) \delta 4.17(\mathrm{br} \mathrm{s}, 2 \mathrm{H}), 6.67(\mathrm{~d}, J$ $=8.7 \mathrm{~Hz}, 1 \mathrm{H}), 7.24(\mathrm{dd}, J=8.7,2.4 \mathrm{~Hz}, 1 \mathrm{H}), 7.40(\mathrm{~d}, J=2.4 \mathrm{~Hz}, 1 \mathrm{H})$; ${ }^{13} \mathrm{C} \mathrm{NMR}\left(126 \mathrm{MHz}, \mathrm{CDCl}_{3}\right) \delta 114.8\left(\mathrm{C}, \mathrm{q},{ }^{2} J_{\mathrm{CF}}=30.8 \mathrm{~Hz}\right), 118.4$ $(\mathrm{CH}), 122.4(\mathrm{C}), 124.1\left(\mathrm{C}, \mathrm{q},{ }^{1} J_{\mathrm{CF}}=272.4 \mathrm{~Hz}\right), 126.3\left(\mathrm{CH}, \mathrm{q},{ }^{3} J_{\mathrm{CF}}=\right.$ $5.5 \mathrm{~Hz}), 132.8(\mathrm{CH}), 143.1(\mathrm{C})$; MS (EI) $\mathrm{m} / z 195\left(\mathrm{M}^{+}, 59\right), 175$ (45), 148 (36), 107 (19), 84 (100), 77 (46).

2-Chloro-4-nitroaniline $(7 k))^{33}$ The reaction was performed as described in general procedure A using 4-nitroaniline $(6 \mathbf{k})(138 \mathrm{mg}$, $1.00 \mathrm{mmol})$. The reaction mixture was heated to $60{ }^{\circ} \mathrm{C}$ for $24 \mathrm{~h}$. Purification by flash column chromatography (petroleum ether/ethyl acetate, 9:1) gave 2-chloro-4-nitroaniline (7k) (153 mg, 89\%) as a yellow solid: $\mathrm{mp} 98-100{ }^{\circ} \mathrm{C}$ (lit. $\left.{ }^{33} \mathrm{mp} 99-101{ }^{\circ} \mathrm{C}\right) ;{ }^{1} \mathrm{H}$ NMR (500 $\left.\mathrm{MHz}, \mathrm{CDCl}_{3}\right) \delta 4.85$ (br s, $\left.2 \mathrm{H}\right), 6.75(\mathrm{~d}, J=8.9 \mathrm{~Hz}, 1 \mathrm{H}), 7.98(\mathrm{dd}, J=$ $8.9,2.5 \mathrm{~Hz}, 1 \mathrm{H}), 8.19(\mathrm{~d}, J=2.5 \mathrm{~Hz}, 1 \mathrm{H}) ;{ }^{13} \mathrm{C} \mathrm{NMR}(126 \mathrm{MHz}$, $\left.\mathrm{CDCl}_{3}\right) \delta 113.7(\mathrm{CH}), 117.7(\mathrm{C}), 124.4(\mathrm{CH}), 126.0(\mathrm{CH}), 138.8$ (C), 148.9 (C); MS (EI) $m / z 172\left(\mathrm{M}^{+}, 100\right), 142$ (60), 126 (28), 99 (21), 90 (70), 63 (29).

4-Amino-3-chlorobenzonitrile (7) ${ }^{34}{ }^{34}$ The reaction was performed as described in general procedure A using 4-aminobenzonitrile (61) (118 mg, $1.00 \mathrm{mmol}$ ), iron(III) chloride $(8.00 \mathrm{mg}, 0.0500 \mathrm{mmol})$, and 
[BMIM] NTf $2(44.0 \mu \mathrm{L}, 0.150 \mathrm{mmol})$ in tetrahydrofuran $(600 \mu \mathrm{L})$. The reaction mixture was heated to $70{ }^{\circ} \mathrm{C}$ for $24 \mathrm{~h}$. Purification by flash column chromatography (petroleum ether/ethyl acetate, 9:1) gave 4-amino-3-chlorobenzonitrile (7l) (142 $\mathrm{mg}, 93 \%)$ as a white solid: $\mathrm{mp} 102-104{ }^{\circ} \mathrm{C}\left(\mathrm{lit} .{ }^{34} \mathrm{mp} 105.7-107.9{ }^{\circ} \mathrm{C}\right) ;{ }^{1} \mathrm{H}$ NMR (400 $\left.\mathrm{MHz}, \mathrm{CDCl}_{3}\right) \delta 4.65(\mathrm{br} \mathrm{s}, 2 \mathrm{H}), 6.75(\mathrm{~d}, J=8.4 \mathrm{~Hz}, 1 \mathrm{H}), 7.31(\mathrm{dd}, J=$ 8.4, $1.8 \mathrm{~Hz}, 1 \mathrm{H}), 7.50(\mathrm{~d}, J=1.8 \mathrm{~Hz}, 1 \mathrm{H}) ;{ }^{13} \mathrm{C}$ NMR $(101 \mathrm{MHz}$, $\left.\mathrm{CDCl}_{3}\right) \delta 100.7(\mathrm{C}), 115.1(\mathrm{CH}), 118.5(\mathrm{C}), 119.0(\mathrm{C}), 132.0(\mathrm{CH})$, $133.3(\mathrm{CH}), 147.2(\mathrm{C})$; MS (EI) $m / z 152\left(\mathrm{M}^{+}, 100\right), 125(45), 117$ (46), 90 (47), 76 (40), 63 (45).

4-Chloroacetanilide $(7 \mathrm{~m}))^{35}$ The reaction was performed as described in general procedure A using acetanilide $(6 \mathrm{~m})(135 \mathrm{mg}$, $1.00 \mathrm{mmol}$ ). The reaction mixture was heated to $60{ }^{\circ} \mathrm{C}$ for $24 \mathrm{~h}$. Purification by flash column chromatography (petroleum ether/ethyl acetate, 4:1) gave 4-chloroacetanilide $(7 \mathbf{m})(132 \mathrm{~g}, 78 \%)$ as a white solid: mp $174-176^{\circ} \mathrm{C}\left(\right.$ lit. $\left.^{35} \mathrm{mp} 175-178^{\circ} \mathrm{C}\right) ;{ }^{1} \mathrm{H}$ NMR $(500 \mathrm{MHz}$, $\left.\mathrm{CDCl}_{3}\right) \delta 2.17(\mathrm{~s}, 3 \mathrm{H}), 7.21-7.30(\mathrm{~m}, 3 \mathrm{H}), 7.42-7.48(\mathrm{~m}, 2 \mathrm{H}) ;{ }^{13} \mathrm{C}$ NMR $\left(126 \mathrm{MHz}, \mathrm{CDCl}_{3}\right) \delta 24.6\left(\mathrm{CH}_{3}\right), 121.0(2 \times \mathrm{CH}), 129.0(2 \times$ $\mathrm{CH}), 129.3$ (C), 136.4 (C), 168.2 (C); MS (ESI) $\mathrm{m} / z 192\left(\mathrm{MNa}^{+}\right.$, 100).

1-Chloro-4-methoxynaphthalene $(7 n) .^{36}$ The reaction was performed as described in general procedure A using 1-methoxynaphthalene (6n) $(144 \mu \mathrm{L}, 1.00 \mathrm{mmol})$. The reaction mixture was heated to $60{ }^{\circ} \mathrm{C}$ for $18 \mathrm{~h}$. Purification by flash column chromatography (petroleum ether/ethyl acetate, 19:1) gave 1-chloro-4-methoxynaphthalene $(7 \mathbf{n})(175 \mathrm{mg}, 91 \%)$ as a colorless oil. Spectroscopic data were consistent with the literature: ${ }^{36}{ }^{1} \mathrm{H}$ NMR $\left(400 \mathrm{MHz}, \mathrm{CDCl}_{3}\right) \delta 4.00$ $(\mathrm{s}, 3 \mathrm{H}), 6.73(\mathrm{~d}, J=8.3 \mathrm{~Hz}, 1 \mathrm{H}), 7.46(\mathrm{~d}, J=8.3 \mathrm{~Hz}, 1 \mathrm{H}), 7.54$ (ddd, $J$ $=8.3,6.9,1.3 \mathrm{~Hz}, 1 \mathrm{H}), 7.62(\mathrm{ddd}, J=8.4,6.9,1.4 \mathrm{~Hz}, 1 \mathrm{H}), 8.20(\mathrm{br} \mathrm{d}$, $J=8.4 \mathrm{~Hz}, 1 \mathrm{H}), 8.29($ br d, $J=8.3 \mathrm{~Hz}, 1 \mathrm{H}) ;{ }^{13} \mathrm{C}$ NMR $(101 \mathrm{MHz}$, $\left.\mathrm{CDCl}_{3}\right) \delta 55.7\left(\mathrm{CH}_{3}\right), 103.8(\mathrm{CH}), 122.4(\mathrm{CH}), 123.2(\mathrm{C}), 124.2$ $(\mathrm{CH}), 125.7(\mathrm{CH}), 125.9(\mathrm{CH}), 126.6(\mathrm{C}), 127.5(\mathrm{CH}), 131.3(\mathrm{C})$, 154.6 (C); MS (EI) $m / z 192\left(\mathrm{M}^{+}, 100\right), 177$ (52), 149 (66), 114 (14), $84(46)$.

1-Chloro-2-methoxynaphthalene (7o). ${ }^{11 a}$ The reaction was performed as described in general procedure A using 2-methoxynaphthalene $(60)(158 \mathrm{mg}, 1.00 \mathrm{mmol})$, iron(III) chloride $(8.00 \mathrm{mg}$, $0.0500 \mathrm{mmol})$, and [BMIM] NTf $2(44.0 \mu \mathrm{L}, 0.150 \mathrm{mmol})$ in tetrahydrofuran $(600 \mu \mathrm{L})$. The reaction mixture was heated to 70 ${ }^{\circ} \mathrm{C}$ for $18 \mathrm{~h}$. Purification by flash column chromatography (petroleum ether/ethyl acetate, 19:1) gave 1-chloro-2-methoxynaphthalene (7n) (173 mg, 90\%) as a white solid: $\mathrm{mp} 64-66^{\circ} \mathrm{C}\left(\right.$ lit. $\left.{ }^{11 \mathrm{a}} \mathrm{mp} 68-69^{\circ} \mathrm{C}\right)$; ${ }^{1} \mathrm{H} \mathrm{NMR}\left(400 \mathrm{MHz}, \mathrm{CDCl}_{3}\right) \delta 4.03(\mathrm{~s}, 3 \mathrm{H}), 7.29(\mathrm{~d}, J=9.0 \mathrm{~Hz}, 1 \mathrm{H})$, 7.41 (ddd, $J=8.2,6.8,1.2 \mathrm{~Hz}, 1 \mathrm{H}), 7.58$ (ddd, $J=8.4,6.8,1.2 \mathrm{~Hz}$, $1 \mathrm{H}), 7.77$ (d, $J=9.0 \mathrm{~Hz}, 1 \mathrm{H}), 7.80(\mathrm{br} \mathrm{d}, J=8.2 \mathrm{~Hz}, 1 \mathrm{H}), 8.21-8.26$ $(\mathrm{m}, 1 \mathrm{H}) ;{ }^{13} \mathrm{C}$ NMR $\left(101 \mathrm{MHz}, \mathrm{CDCl}_{3}\right) \delta 57.1\left(\mathrm{CH}_{3}\right), 113.8(\mathrm{CH})$, $117.0(\mathrm{C}), 123.6(\mathrm{CH}), 124.4(\mathrm{CH}), 127.6(\mathrm{CH}), 128.1(2 \times \mathrm{CH})$, 129.6 (C), 132.0 (C), 152.7 (C); MS (EI) $\mathrm{m} / z 192\left(\mathrm{M}^{+}, 27\right), 149$ (25), 97 (18), 84 (91), 66 (100), 57 (43).

1-Chloro-2-naphthol (7p). ${ }^{37}$ The reaction was performed as described in general procedure A using 2-naphthol (6p) (144 mg, $1.00 \mathrm{mmol}$ ). The reaction mixture was heated to $60{ }^{\circ} \mathrm{C}$ for $18 \mathrm{~h}$. Purification by flash column chromatography (petroleum ether/ethyl acetate, 9:1) gave 1-chloro-2-naphthol (7p) (174 mg, 97\%) as an offwhite solid: mp $64-66{ }^{\circ} \mathrm{C}$ (lit. $\left.{ }^{37} \mathrm{mp} 66^{\circ} \mathrm{C}\right)$; ${ }^{1} \mathrm{H}$ NMR $(500 \mathrm{MHz}$, $\left.\mathrm{CDCl}_{3}\right) \delta 5.87(\mathrm{~s}, 1 \mathrm{H}), 7.26(\mathrm{~d}, J=8.9 \mathrm{~Hz}, 1 \mathrm{H}), 7.40(\mathrm{ddd}, J=8.1$, 6.8, 1.1 Hz, 1H), 7.57 (ddd, $J=8.5,6.8,1.2 \mathrm{~Hz}, 1 \mathrm{H}), 7.71(\mathrm{~d}, J=8.9$ $\mathrm{Hz}, 1 \mathrm{H}), 7.79($ br d, $J=8.1 \mathrm{~Hz}, 1 \mathrm{H}), 8.06($ br d, $J=8.5 \mathrm{~Hz}, 1 \mathrm{H}) ;{ }^{13} \mathrm{C}$ NMR (126 MHz, $\left.\mathrm{CDCl}_{3}\right) \delta 113.4(\mathrm{C}), 117.3(\mathrm{CH}), 122.8(\mathrm{CH})$, $124.2(\mathrm{CH}), 127.6(\mathrm{CH}), 128.2(\mathrm{CH}), 128.5(\mathrm{CH}), 129.5(\mathrm{C}), 131.1$ (C), 149.4 (C); MS (ESI) $m / z 177\left([\mathrm{M}-\mathrm{H}]^{-}, 100\right)$.

5-Chloro-2,3-dihydrobenzofuran $(7 q){ }^{38}$ The reaction was performed as described in general procedure A using 2,3-dihydrobenzofuran $(6 q)(113 \mu \mathrm{L}, 1.00 \mathrm{mmol})$. The reaction mixture was heated to $60{ }^{\circ} \mathrm{C}$ for $18 \mathrm{~h}$. Purification by flash column chromatography (petroleum ether/ethyl acetate, 9:1) gave 5-chloro-2,3-dihydrobenzofuran $(7 \mathbf{q})(133 \mathrm{mg}, 86 \%)$ as a colorless oil. Spectroscopic data were consistent with the literature: ${ }^{38}{ }^{1} \mathrm{H}$ NMR $\left(500 \mathrm{MHz}, \mathrm{CDCl}_{3}\right) \delta 3.20$ $(\mathrm{t}, J=8.7 \mathrm{~Hz}, 2 \mathrm{H}), 4.58(\mathrm{t}, J=8.7 \mathrm{~Hz}, 2 \mathrm{H}), 6.69(\mathrm{~d}, J=8.4 \mathrm{~Hz}, 1 \mathrm{H})$, $7.05(\mathrm{dd}, J=8.4,2.2 \mathrm{~Hz}, 1 \mathrm{H}), 7.13-7.16(\mathrm{~m}, 1 \mathrm{H}, \mathrm{m}) ;{ }^{13} \mathrm{C}$ NMR $(126$
$\left.\mathrm{MHz}, \mathrm{CDCl}_{3}\right) \delta 29.7\left(\mathrm{CH}_{2}\right), 71.6\left(\mathrm{CH}_{2}\right), 110.2(\mathrm{CH}), 125.0(\mathrm{C}$ and $\mathrm{CH}), 127.8(\mathrm{CH}), 128.9$ (C), 158.7 (C); MS (EI) m/z $154\left(\mathrm{M}^{+}, 80\right)$, 91 (41), 84 (100).

2,4-Dichlorophenol (2). ${ }^{39}$ The reaction was performed as described in general procedure A using phenol (6f) $(94.0 \mathrm{mg}, 1.00 \mathrm{mmol})$, NCS $(280 \mathrm{mg}, 2.10 \mathrm{mmol})$ in tetrahydrofuran $(1.2 \mathrm{~mL})$. The reaction mixture was heated to $60{ }^{\circ} \mathrm{C}$ for $48 \mathrm{~h}$. Purification by flash column chromatography (petroleum ether/diethyl ether 19:1) gave 2,4dichlorophenol (2) (131 $\mathrm{mg}, 81 \%)$ as a light orange solid: $\mathrm{mp} 40-$ $42{ }^{\circ} \mathrm{C}\left(\right.$ lit. $\left.^{39} \mathrm{mp} 43-44{ }^{\circ} \mathrm{C}\right) ;{ }^{1} \mathrm{H}$ NMR $\left(400 \mathrm{MHz}, \mathrm{CDCl}_{3}\right) \delta 5.49(\mathrm{~s}$, $1 \mathrm{H}), 6.95(\mathrm{~d}, J=8.7 \mathrm{~Hz}, 1 \mathrm{H}), 7.15(\mathrm{dd}, J=8.7,2.5 \mathrm{~Hz}, 1 \mathrm{H}), 7.33$ (d, $J$ $=2.5 \mathrm{~Hz}, 1 \mathrm{H}) ;{ }^{13} \mathrm{C} \mathrm{NMR}\left(101 \mathrm{MHz}, \mathrm{CDCl}_{3}\right) \delta 117.3(\mathrm{CH}), 120.6$ (C), 125.8 (C), $128.7(2 \times \mathrm{CH}), 150.4$ (C); MS (EI) $\mathrm{m} / z 162\left(\mathrm{M}^{+}\right.$, 100), 153 (21), 136 (18), 107 (46), 89 (38).

5-Chloro-2-methoxybenzaldehyde (7r). ${ }^{40}$ The reaction was performed as described in general procedure B using 2-methoxybenzaldehyde $(6 \mathbf{r})(60 \mathrm{mg}, 0.44 \mathrm{mmol})$. The reaction mixture was heated to $70{ }^{\circ} \mathrm{C}$ for $18 \mathrm{~h}$. Purification by flash column chromatography (petroleum ether/ethyl acetate, 9:1) gave 5-chloro-2-methoxybenzaldehyde $(7 \mathbf{r})(51 \mathrm{mg}, 68 \%)$ as a white solid: $\mathrm{mp} 78-79{ }^{\circ} \mathrm{C}$ (lit. ${ }^{40} \mathrm{mp}$ $\left.80-81{ }^{\circ} \mathrm{C}\right) ;{ }^{1} \mathrm{H}$ NMR $\left(400 \mathrm{MHz}, \mathrm{CDCl}_{3}\right) \delta 3.93(\mathrm{~s}, 3 \mathrm{H}), 6.95(\mathrm{~d}, J=$ $8.9 \mathrm{~Hz}, 1 \mathrm{H}), 7.50(\mathrm{dd}, J=8.9,2.8 \mathrm{~Hz}, 1 \mathrm{H}), 7.79(\mathrm{~d}, J=2.8 \mathrm{~Hz}, 1 \mathrm{H})$, $10.41(\mathrm{~s}, 1 \mathrm{H}) ;{ }^{13} \mathrm{C}$ NMR $\left(126 \mathrm{MHz}, \mathrm{CDCl}_{3}\right) \delta 56.0\left(\mathrm{CH}_{3}\right), 113.3$ $(\mathrm{CH}), 125.7(\mathrm{C}), 126.4(\mathrm{C}), 128.0(\mathrm{CH}), 135.4(\mathrm{CH}), 160.3(\mathrm{C})$, $188.5(\mathrm{CH}) ; \mathrm{MS}(\mathrm{EI}) \mathrm{m} / z 170\left(\mathrm{M}^{+}, 17\right), 153(6), 84$ (100), 49 (79), $44(32)$.

3-Chloro-4-methoxybenzaldehyde (7s). ${ }^{41}$ The reaction was performed as described in general procedure B using 4-methoxybenzaldehyde (6s) $(122 \mu \mathrm{L}, 1.00 \mathrm{mmol})$ and NCS (160 mg, 1.20 $\mathrm{mmol})$. The reaction mixture was heated to $70{ }^{\circ} \mathrm{C}$ for $18 \mathrm{~h}$. Purification by flash column chromatography (petroleum ether/ethyl acetate 19:1) gave 3-chloro-4-methoxybenzaldehyde (7s) (141 mg, $83 \%)$ as a pale yellow solid: $\mathrm{mp} 52-54{ }^{\circ} \mathrm{C}\left(\right.$ lit. $\left.^{41} \mathrm{mp} 53-55^{\circ} \mathrm{C}\right) ;{ }^{1} \mathrm{H}$ NMR $\left(400 \mathrm{MHz}, \mathrm{CDCl}_{3}\right) \delta 3.99(\mathrm{~s}, 3 \mathrm{H}), 7.04(\mathrm{~d}, J=8.5 \mathrm{~Hz}, 1 \mathrm{H})$, $7.77(\mathrm{dd}, J=8.5,2.0 \mathrm{~Hz}, 1 \mathrm{H}), 7.91(\mathrm{~d}, J=2.0 \mathrm{~Hz}, 1 \mathrm{H}), 9.85(\mathrm{~s}, 1 \mathrm{H})$; ${ }^{13} \mathrm{C}$ NMR $\left(101 \mathrm{MHz}, \mathrm{CDCl}_{3}\right) \delta 56.5\left(\mathrm{CH}_{3}\right), 111.7(\mathrm{CH}), 123.7(\mathrm{C})$, $130.3(\mathrm{C}), 130.5(\mathrm{CH}), 131.2(\mathrm{CH}), 159.8(\mathrm{C}), 189.6(\mathrm{CH})$; MS (EI) m/z $169\left(\mathrm{M}^{+}, 100\right), 143$ (13), 126 (15), 115 (19), 99 (14), 83 (16), 75 (12), 63 (26).

3-Chloro-4-methoxybenzoic Acid (7t). ${ }^{42}$ The reaction was performed as described in general procedure B using 4-methoxybenzoic acid $(6 \mathrm{t})(76 \mathrm{mg}, 0.50 \mathrm{mmol})$. The reaction mixture was heated to $70{ }^{\circ} \mathrm{C}$ for $24 \mathrm{~h}$. Purification by flash column chromatography (petroleum ether/ethyl acetate, 17:3) gave 3-chloro-4-methoxybenzoic acid $(7 t)(86 \mathrm{mg}, 92 \%)$ as a white solid: $\mathrm{mp} 208-210^{\circ} \mathrm{C}$ (lit. ${ }^{42} \mathrm{mp}$ 211-214 $\left.{ }^{\circ} \mathrm{C}\right) ;{ }^{1} \mathrm{H}$ NMR $\left(400 \mathrm{MHz}, \mathrm{CDCl}_{3}\right) \delta 3.99$ (s, 3H), 6.99 (d, $J$ $=8.7 \mathrm{~Hz}, 1 \mathrm{H}), 8.02(\mathrm{dd}, J=8.7,2.1 \mathrm{~Hz}, 1 \mathrm{H}), 8.13(\mathrm{~d}, J=2.1 \mathrm{~Hz}, 1 \mathrm{H})$; ${ }^{13} \mathrm{C}$ NMR $\left(101 \mathrm{MHz}, \mathrm{CDCl}_{3}\right) \delta 56.6\left(\mathrm{CH}_{3}\right), 111.5(\mathrm{CH}), 122.4(\mathrm{C})$, 122.9 (C), $130.8(\mathrm{CH}), 132.5$ (CH), 159.5 (C), 170.0 (C); MS (EI) $\mathrm{m} / z 186\left(\mathrm{M}^{+}, 83\right), 169$ (41), 115 (13), 83 (100), 63 (11).

2-Chloro-4-nitrophenol (1). ${ }^{43}$ The reaction was performed as described in general procedure B using 4-nitrophenol $(70 \mathrm{mg}, 0.50$ $\mathrm{mmol})$. The reaction mixture was heated to $70{ }^{\circ} \mathrm{C}$ for $48 \mathrm{~h}$. Purification by flash column chromatography (petroleum ether/ethyl acetate 3:1) gave 2-chloro-4-nitrophenol (1) (64 $\mathrm{mg}, 74 \%$ ) as a yellow solid: mp $108-110^{\circ} \mathrm{C}\left(\right.$ lit. $\left.{ }^{43} \mathrm{mp} 110-112{ }^{\circ} \mathrm{C}\right) ;{ }^{1} \mathrm{H}$ NMR $(400 \mathrm{MHz}$, $\left.\mathrm{CDCl}_{3}\right) \delta 6.34(\mathrm{~s}, 1 \mathrm{H}), 7.13(\mathrm{~d}, J=9.0 \mathrm{~Hz}, 1 \mathrm{H}), 8.12(\mathrm{dd}, J=9.0,2.7$ $\mathrm{Hz}, 1 \mathrm{H}), 8.29(\mathrm{~d}, J=2.7 \mathrm{~Hz}, 1 \mathrm{H}) ;{ }^{13} \mathrm{C}$ NMR $\left(101 \mathrm{MHz}, \mathrm{CDCl}_{3}\right) \delta$ $116.4(\mathrm{CH}), 120.5(\mathrm{C}), 124.7(\mathrm{CH}), 125.5(\mathrm{CH}), 141.7(\mathrm{C}), 157.0$ (C); MS (EI) m/z $173\left(\mathrm{M}^{+}, 100\right), 143$ (39), 107 (21), 99 (37), 84 (65), 63 (36).

$N$-(2-Acetyl-4-chlorophenyl)acetamide (7u). The reaction was performed as described in general procedure B using $\mathrm{N}$-(2acetylphenyl)acetamide $(6 \mathbf{u})(99 \mathrm{mg}, 0.50 \mathrm{mmol})$. The reaction mixture was heated to $70{ }^{\circ} \mathrm{C}$ for $48 \mathrm{~h}$. Purification by flash column chromatography (petroleum ether/diethyl ether, 9:1) gave $\mathrm{N}$-(2acetyl-4-chlorophenyl)acetamide $(7 \mathbf{u})(101 \mathrm{mg}, 95 \%)$ as a white solid: $\mathrm{mp} 126-128^{\circ} \mathrm{C}$; IR (neat) $3218,2359,1685,1657,1588,1501,1400$, $1360,1311,1286,1246,1224,828,773,637 \mathrm{~cm}^{-1}$; ${ }^{1} \mathrm{H}$ NMR (400 $\left.\mathrm{MHz} \mathrm{CDCl}_{3}\right) \delta 2.22(\mathrm{~s}, 3 \mathrm{H}), 2.65(\mathrm{~s}, 3 \mathrm{H}), 7.49(\mathrm{dd}, J=9.1,2.5 \mathrm{~Hz}$, 
$1 \mathrm{H}), 7.82(\mathrm{~d}, J=2.5 \mathrm{~Hz}, 1 \mathrm{H}), 8.72(\mathrm{~d}, J=9.1 \mathrm{~Hz}, 1 \mathrm{H}), 11.56($ br s, $1 \mathrm{H}) ;{ }^{13} \mathrm{C} \mathrm{NMR}\left(101 \mathrm{MHz}, \mathrm{CDCl}_{3}\right) \delta 25.6\left(\mathrm{CH}_{3}\right), 28.7\left(\mathrm{CH}_{3}\right), 122.4$ $(\mathrm{CH}), 122.9(\mathrm{C}), 127.3(\mathrm{C}), 131.2(\mathrm{CH}), 135.0(\mathrm{CH}), 139.7(\mathrm{C})$, 169.6 (C), 201.9 (C); MS (EI) $m / z 211\left(\mathrm{M}^{+}, 44\right), 169$ (97), 154 (100); HRMS (EI) calcd for $\mathrm{C}_{10} \mathrm{H}_{10}{ }^{35} \mathrm{ClNO}_{2}\left(\mathrm{M}^{+}\right)$211.0400, found 211.0402 .

3,5-Dichloro-4-hydroxybenzonitrile (5). The reaction was performed as described in general procedure B using 4-hydroxybenzonitrile $(60.0 \mathrm{mg}, 0.500 \mathrm{mmol})$ and NCS $(147 \mathrm{mg}, 1.10 \mathrm{mmol})$. The reaction mixture was heated to $70{ }^{\circ} \mathrm{C}$ and stirred for $36 \mathrm{~h}$. Purification by flash column chromatography (petroleum ether/ethyl acetate, 7:3) gave 3,5-dichloro-4-hydroxybenzonitrile (5) $(68 \mathrm{mg}, 73 \%)$ as a white solid: $\mathrm{mp} 128-130^{\circ} \mathrm{C}$; IR (neat) $3255,2924,2243,1483,1302,1150$, $909 \mathrm{~cm}^{-1}$; ${ }^{1} \mathrm{H}$ NMR $\left(400 \mathrm{MHz}, \mathrm{CDCl}_{3}\right) \delta 6.48(\mathrm{br} \mathrm{s}, 1 \mathrm{H}), 7.59(\mathrm{~s}$, $2 \mathrm{H}) ;{ }^{13} \mathrm{C}$ NMR (101 MHz, CDCl $\left.{ }_{3}\right) \delta 105.4(\mathrm{C}), 116.7$ (C), $122.3(2$ $\times \mathrm{C}), 132.2(2 \times \mathrm{CH}), 152.3(\mathrm{C})$; MS (EI) $\mathrm{m} / z 187\left(\mathrm{M}^{+}, 100\right)$; HRMS (EI) calcd for $\mathrm{C}_{7} \mathrm{H}_{3}{ }^{35} \mathrm{Cl}_{2} \mathrm{NO}\left(\mathrm{M}^{+}\right)$186.9592, found 186.9583 .

4-Bromo-2-chloroanisole (8). ${ }^{44} \mathrm{~N}$-Bromosuccinimide $(178 \mathrm{mg}$, $1.00 \mathrm{mmol}$ ) was added to a solution of iron(III) chloride $(8.00 \mathrm{mg}$, $0.0500 \mathrm{mmol})$ in [BMIM] NTf $\mathrm{N}_{2}(0.40 \mathrm{~mL})$ under an atmosphere of air. The mixture was stirred at room temperature for $0.5 \mathrm{~h}$ before anisole (6a) $(108 \mu \mathrm{L}, 1.00 \mathrm{mmol})$ in acetonitrile $(0.10 \mathrm{~mL})$ was added. The reaction mixture was heated to $40{ }^{\circ} \mathrm{C}$ for $1.5 \mathrm{~h}$. The reaction mixture was cooled to room temperature, and $\mathrm{N}$-chlorosuccinimide $(160 \mathrm{mg}$, $1.20 \mathrm{mmol}$ ) was then added. The reaction mixture was heated to 70 ${ }^{\circ} \mathrm{C}$ for $24 \mathrm{~h}$. The reaction was worked up according to general chlorination procedure B. Purification by flash column chromatography (petroleum ether/ethyl acetate, 19:1) gave 4-bromo-2chloroanisole $(8)(197 \mathrm{mg}, 89 \%)$ as a pale yellow solid: $\mathrm{mp}$ 66-68 ${ }^{\circ} \mathrm{C}\left(\right.$ lit. $\left.{ }^{44} \mathrm{mp} 66.5-68.2{ }^{\circ} \mathrm{C}\right) ;{ }^{1} \mathrm{H}$ NMR $\left(400 \mathrm{MHz}, \mathrm{CDCl}_{3}\right) \delta 3.88(\mathrm{~s}$, $3 \mathrm{H}), 6.79(\mathrm{~d}, J=8.8 \mathrm{~Hz}, 1 \mathrm{H}), 7.33(\mathrm{dd}, J=8.8,2.4 \mathrm{~Hz}, 1 \mathrm{H}), 7.50(\mathrm{~d}, J$ $=2.4 \mathrm{~Hz}, 1 \mathrm{H}) ;{ }^{13} \mathrm{C}$ NMR $\left(101 \mathrm{MHz}^{\mathrm{CDCl}} \mathrm{CDC}_{3} \delta 56.3\left(\mathrm{CH}_{3}\right), 112.5\right.$ (C), $113.3(\mathrm{CH}), 123.7(\mathrm{C}), 130.5(\mathrm{CH}), 132.7(\mathrm{CH}), 154.4(\mathrm{C})$; MS (EI) $m / z 222\left(\mathrm{M}^{+}, 86\right), 179$ (100), 126 (36).

2-Bromo-4-chloroanisole (9). ${ }^{45}$ The reaction was performed as described in general procedure B using anisole (6a) $(108 \mu \mathrm{L}, 1.00$ $\mathrm{mmol})$. The reaction mixture was heated to $60{ }^{\circ} \mathrm{C}$ for $6 \mathrm{~h}$. On completion of the chlorination step, the reaction mixture was cooled to room temperature and $N$-bromosuccinimide $(178 \mathrm{mg}, 1.00 \mathrm{mmol})$ was then added. The reaction mixture was heated to $40{ }^{\circ} \mathrm{C}$ for $18 \mathrm{~h}$. Purification by flash column chromatography (petroleum ether/ethyl acetate, 17:3) gave 2-bromo-4-chloroanisole (9) (178 mg, 81\%) as a colorless oil. Spectroscopic data were consistent with the literature: ${ }^{45}$ ${ }^{1} \mathrm{H}$ NMR $\left(500 \mathrm{MHz}, \mathrm{CDCl}_{3}\right) \delta 3.88(\mathrm{~s}, 3 \mathrm{H}), 6.82(\mathrm{~d}, J=8.8 \mathrm{~Hz}, 1 \mathrm{H})$, $7.24(\mathrm{dd}, J=8.8,2.5 \mathrm{~Hz}, 1 \mathrm{H}), 7.54(\mathrm{~d}, J=2.5 \mathrm{~Hz}, 1 \mathrm{H}) ;{ }^{13} \mathrm{C} \mathrm{NMR}$ $\left(126 \mathrm{MHz}, \mathrm{CDCl}_{3}\right) \delta 56.6\left(\mathrm{CH}_{3}\right), 112.3(\mathrm{C}), 112.7(\mathrm{CH}), 126.1(\mathrm{C})$, $128.4(\mathrm{CH}), 132.9(\mathrm{CH}), 154.9(\mathrm{C})$; MS (EI) $\mathrm{m} / z 222\left(\mathrm{M}^{+}, 100\right)$, 207 (50), 179 (36), 126 (10), 75 (12), 63 (21).

$\mathrm{N}$-(5'-Chloro-2'-methoxyphenyl)-4-chlorobenzenesulfonamide (10). ${ }^{22}$ To a solution of 2-bromo-4-chloroanisole $(9)(110 \mathrm{mg}, 0.500$ $\mathrm{mmol})$ in toluene $(0.50 \mathrm{~mL})$ were added 4-chlorobenzenesulfonamide (115 mg, $0.600 \mathrm{mmol})$, copper(I) iodide $(10.0 \mathrm{mg}, 0.0500 \mathrm{mmol})$, $N, N^{\prime}$-dimethylethylenediamine $(11.0 \mu \mathrm{L}, 0.100 \mathrm{mmol})$, cesium carbonate $(326 \mathrm{mg}, 1.00 \mathrm{mmol})$, and water $(0.30 \mathrm{~mL})$. The reaction mixture was degassed under argon for $0.1 \mathrm{~h}$ and then heated to $150{ }^{\circ} \mathrm{C}$ for $24 \mathrm{~h}$. The reaction mixture was then cooled to room temperature, diluted with ethyl acetate $(10 \mathrm{~mL})$, and washed with $1 \mathrm{M}$ sodium thiosulfate solution $(10 \mathrm{~mL})$ and brine $(10 \mathrm{~mL})$. The organic phase was dried $\left(\mathrm{MgSO}_{4}\right)$, filtered, and concentrated in vacuo. Purification by flash column chromatography (petroleum ether/ethyl acetate, 4:1) gave $\mathrm{N}$-(5'-chloro-2'-methoxyphenyl)-4-chlorobenzenesulfonamide (10) $(107 \mathrm{mg}, 65 \%)$ as a white solid: $\mathrm{mp} 142-144{ }^{\circ} \mathrm{C}$ (lit. ${ }^{22} \mathrm{mp}$ 144-146 $\left.{ }^{\circ} \mathrm{C}\right) ;{ }^{1} \mathrm{H}$ NMR $\left(400 \mathrm{MHz}, \mathrm{CDCl}_{3}\right) \delta 3.66(\mathrm{~s}, 3 \mathrm{H}), 6.67(\mathrm{~d}, J$ $=8.7 \mathrm{~Hz}, 1 \mathrm{H}), 7.01(\mathrm{dd}, J=8.7,2.5 \mathrm{~Hz}, 1 \mathrm{H}), 7.03(\mathrm{br} \mathrm{s}, 1 \mathrm{H}), 7.38-$ $7.42(\mathrm{~m}, 2 \mathrm{H}), 7.53(\mathrm{~d}, J=2.5 \mathrm{~Hz}, 1 \mathrm{H}), 7.69-7.74(\mathrm{~m}, 2 \mathrm{H}) ;{ }^{13} \mathrm{C}$ NMR $\left(101 \mathrm{MHz}, \mathrm{CDCl}_{3}\right) \delta 56.0\left(\mathrm{CH}_{3}\right), 111.5(\mathrm{CH}), 121.0(\mathrm{CH}), 125.3$ (CH), $126.2(\mathrm{C}), 126.5(\mathrm{C}), 128.6(2 \times \mathrm{CH}), 129.2(2 \times \mathrm{CH}), 137.4$ (C), 139.7 (C), 148.0 (C); MS (EI) $m / z 331\left(\mathrm{M}^{+}, 100\right), 296$ (52), 262 (18), 158 (32), 156 (100), 126 (19), 111 (27), 93 (48), 75 (16).
$\mathrm{N}$-(5'-Chloro-2'-methoxyphenyl)benzenesulfonamide (11). ${ }^{23}$ The reaction was performed as described for 4-chloro- $\mathrm{N}-\left(5^{\prime}\right.$-chloro- $2^{\prime}$ methoxyphenyl)benzenesulfonamide (10) using 2-bromo-4-chloroanisole (9) $(221 \mathrm{mg}, 1.00 \mathrm{mmol})$ and benzenesulfonamide (189 mg, 1.20 $\mathrm{mmol}$ ). Purification by flash column chromatography (petroleum ether/ethyl acetate, 7:3) gave $N$-(5'-chloro-2'-methoxyphenyl)benzenesulfonamide (11) (199 mg, 67\%) as a white solid: $\mathrm{mp} 140-$ $142{ }^{\circ} \mathrm{C}$ (lit. $\left.{ }^{23} \mathrm{mp} 140-142{ }^{\circ} \mathrm{C}\right) ;{ }^{1} \mathrm{H}$ NMR $\left(400 \mathrm{MHz}, \mathrm{CDCl}_{3}\right) \delta 3.63$ $(\mathrm{s}, 3 \mathrm{H}), 6.64(\mathrm{~d}, J=8.7 \mathrm{~Hz}, 1 \mathrm{H}), 6.98(\mathrm{dd}, J=8.7,2.6 \mathrm{~Hz}, 1 \mathrm{H}), 7.02$ (br s, $1 \mathrm{H}), 7.40-7.47(\mathrm{~m}, 2 \mathrm{H}), 7.50-7.53(\mathrm{~m}, 1 \mathrm{H}), 7.55(\mathrm{~d}, J=2.6$ $\mathrm{Hz}, 1 \mathrm{H}), 7.76-7.82(\mathrm{~m}, 2 \mathrm{H}) ;{ }^{13} \mathrm{C}$ NMR $\left(101 \mathrm{MHz}, \mathrm{CDCl}_{3}\right) \delta 56.0$ $\left(\mathrm{CH}_{3}\right), 111.5(\mathrm{CH}), 120.8(\mathrm{CH}), 124.9(\mathrm{CH}), 126.1(\mathrm{C}), 126.9(\mathrm{C})$, $127.2(2 \times \mathrm{CH}), 128.9(2 \times \mathrm{CH}), 133.1(\mathrm{CH}), 139.0(\mathrm{C}), 148.0(\mathrm{C})$; MS (EI) $m / z 297\left(\mathrm{M}^{+}, 79\right), 156$ (100), 93 (70), 77 (40), 51 (27).

$N$-(5'-Chloro-2'-methoxyphenyl)- $N$-methylbenzenesulfonamide (12). ${ }^{23}$ To a solution of $N$-( $5^{\prime}$-chloro-2'-methoxyphenyl)benzenesulfonamide (11) (38 mg, $0.13 \mathrm{mmol})$ in DMF $(0.7 \mathrm{~mL})$ were added potassium carbonate $(53 \mathrm{mg}, 0.38 \mathrm{mmol})$ and iodomethane $(24 \mu \mathrm{L}, 0.38 \mathrm{mmol})$. The reaction mixture was stirred at room temperature for $2 \mathrm{~h}$. The reaction mixture was then quenched with water $(2 \mathrm{~mL})$ and extracted with diethyl ether $(3 \times 3 \mathrm{~mL})$. The organic layers were combined, dried $\left(\mathrm{MgSO}_{4}\right)$, filtered, and concentrated in vacuo. Purification by flash column chromatography (petroleum ether/ethyl acetate, $4: 1)$ gave $N$-(5'-chloro-2'-methoxyphenyl)- $N$-methylbenzenesulfonamide (12) (37 mg, 93\%) as a colorless oil. Spectroscopic data were consistent with the literature: ${ }^{23}$ ${ }^{1} \mathrm{H} \operatorname{NMR}\left(500 \mathrm{MHz}, \mathrm{CDCl}_{3}\right) \delta 3.18(\mathrm{~s}, 3 \mathrm{H}), 3.36(\mathrm{~s}, 3 \mathrm{H}), 6.71(\mathrm{~d}, J=$ $8.8 \mathrm{~Hz}, 1 \mathrm{H}), 7.23(\mathrm{dd}, J=8.8,2.7 \mathrm{~Hz}, 1 \mathrm{H}), 7.29(\mathrm{~d}, J=2.7 \mathrm{~Hz}, 1 \mathrm{H})$, 7.44-7.50 (m, 2H), 7.53-7.59 (m, 1H), 7.66-7.71 (m, 2H); ${ }^{13} \mathrm{C}$ NMR $\left(126 \mathrm{MHz}, \mathrm{CDCl}_{3}\right) \delta 37.8\left(\mathrm{CH}_{3}\right), 55.3\left(\mathrm{CH}_{3}\right), 112.6(\mathrm{CH})$, $125.1(\mathrm{C}), 127.5(2 \times \mathrm{CH}), 128.6(2 \times \mathrm{CH}), 129.4(\mathrm{CH}), 129.9(\mathrm{C})$, $131.8(\mathrm{CH}), 132.4(\mathrm{CH}), 139.2(\mathrm{C}), 155.2(\mathrm{C}) ; \mathrm{MS}(\mathrm{EI}) \mathrm{m} / \mathrm{z} 311$ $\left(\mathrm{M}^{+}, 41\right), 170$ (100), 155 (39), 84 (15), 77 (19).

2-(5-Acetylthien-2-yl)-4-chloroanisole (14). To a solution of 2bromo-4-chloroanisole $(9)(160 \mathrm{mg}, 0.730 \mathrm{mmol})$ in 1,4-dioxane $(8$ $\mathrm{mL})$ were added 5-acetylthiophene-2-boronic acid (148 mg, 0.870 $\mathrm{mmol})$, potassium carbonate $(303 \mathrm{mg}, 2.19 \mathrm{mmol})$, and water $(6.0$ $\mathrm{mL}$ ). The reaction mixture was degassed with argon for $0.1 \mathrm{~h}$. Bis(triphenylphosphine)palladium(II) dichloride $(51.0 \mathrm{mg}, 0.0730$ mmol) was added, and the reaction mixture was heated to $80{ }^{\circ} \mathrm{C}$ for 4 h. The reaction mixture was cooled to room temperature, and the organic solvent was removed under reduced pressure. The residue was dissolved in ethyl acetate $(15 \mathrm{~mL})$, extracted with water $(10 \mathrm{~mL})$, and washed with brine $(10 \mathrm{~mL})$. The combined organic layers were dried $\left(\mathrm{MgSO}_{4}\right)$, filtered, and concentrated in vacuo. Purification by flash column chromatography (petroleum ether/ethyl acetate, 19:1) gave 2(5-acetylthien-2-yl)-4-chloroanisole (14) $(154 \mathrm{mg}, 79 \%)$ as a yellow solid: $\mathrm{mp} 128-130{ }^{\circ} \mathrm{C}$; IR (neat) $2941,1653,1436,1277,1259,1021$, $807,677 \mathrm{~cm}^{-1}$; ${ }^{1} \mathrm{H}$ NMR $\left(500 \mathrm{MHz}, \mathrm{CDCl}_{3}\right) \delta 2.57(\mathrm{~s}, 3 \mathrm{H}), 3.95(\mathrm{~s}$, $3 \mathrm{H}), 6.93(\mathrm{~d}, J=8.8 \mathrm{~Hz}, 1 \mathrm{H}), 7.27(\mathrm{dd}, J=8.8,2.6 \mathrm{~Hz}, 1 \mathrm{H}), 7.47(\mathrm{~d}, J$ $=4.1 \mathrm{~Hz}, 1 \mathrm{H}), 7.64-7.67(\mathrm{~m}, 2 \mathrm{H}) ;{ }^{13} \mathrm{C}$ NMR $\left(126 \mathrm{MHz}, \mathrm{CDCl}_{3}\right) \delta$ $26.8\left(\mathrm{CH}_{3}\right), 55.9\left(\mathrm{CH}_{3}\right), 112.9(\mathrm{CH}), 123.6(\mathrm{C}), 126.0(\mathrm{C}), 126.2$ $(\mathrm{CH}), 128.0(\mathrm{CH}), 129.3(\mathrm{CH}), 132.1(\mathrm{CH}), 143.8(\mathrm{C}), 146.1(\mathrm{C})$, 154.6 (C), 191.1 (C); MS (ESI) $m / z 289\left(\mathrm{MNa}^{+}, 100\right)$; HRMS (ESI) calcd for $\mathrm{C}_{13} \mathrm{H}_{11}{ }^{35} \mathrm{ClNaO}_{2} \mathrm{~S}\left(\mathrm{MNa}^{+}\right) 289.0060$, found 289.0052 .

2-(5-Acetylthien-2-yl)-4-chlorophenol $\quad$ (3). ${ }^{5 b} \quad$ 2-(5-Acetylthien-2yl)-4-chloroanisole (14) $(50.0 \mathrm{mg}, 0.190 \mathrm{mmol})$ was dissolved in dichloromethane $(3.0 \mathrm{~mL})$ and cooled to $-78^{\circ} \mathrm{C}$. Boron tribromide ( $1 \mathrm{M}$ in dichloromethane) $(374 \mu \mathrm{L}, 0.370 \mathrm{mmol})$ was added dropwise, and the reaction mixture was stirred at $-78^{\circ} \mathrm{C}$ for $1 \mathrm{~h}$ before being warmed to room temperature over $5 \mathrm{~h}$. The reaction mixture was quenched by the addition of a saturated solution of sodium bicarbonate $(2 \mathrm{~mL})$ and then extracted with dichloromethane $(4 \times$ $3 \mathrm{~mL})$. The organic layers were combined, dried $\left(\mathrm{MgSO}_{4}\right)$, filtered, and concentrated in vacuo. Purification by flash column chromatography (petroleum ether/ethyl acetate, 4:1) gave 2-(5-acetylthien-2-yl)4-chlorophenol (3) $(41.0 \mathrm{mg}, 87 \%)$ as a yellow solid: $\mathrm{mp} 212-214^{\circ} \mathrm{C}$ (lit. ${ }^{5 \mathrm{~b}} \mathrm{mp} 218^{\circ} \mathrm{C}$ ); ${ }^{1} \mathrm{H}$ NMR (500 MHz, DMSO-d $\left.d_{6}\right) \delta 2.53(\mathrm{~s}, 3 \mathrm{H})$ $7.00(\mathrm{~d}, J=8.7 \mathrm{~Hz}, 1 \mathrm{H}), 7.25$ (dd, $J=8.7,2.6 \mathrm{~Hz}, 1 \mathrm{H}), 7.79(\mathrm{~d}, J=4.1$ $\mathrm{Hz}, 1 \mathrm{H}), 7.84(\mathrm{~d}, J=2.6 \mathrm{~Hz}, 1 \mathrm{H}), 7.89(\mathrm{~d}, J=4.1 \mathrm{~Hz}, 1 \mathrm{H}), 11.00(\mathrm{br}$ 
s, $1 \mathrm{H}) ;{ }^{13} \mathrm{C}$ NMR $\left(126 \mathrm{MHz}\right.$, DMSO- $\left.d_{6}\right) \delta 27.0\left(\mathrm{CH}_{3}\right), 118.5(\mathrm{CH})$, $121.7(\mathrm{C}), 123.7(\mathrm{C}), 126.7(\mathrm{CH}), 127.3(\mathrm{CH}), 129.8(\mathrm{CH}), 133.8$ (CH), 143.5 (C), 146.0 (C), 153.4 (C), 191.5 (C); MS (ESI) $m / z 251$ $\left([\mathrm{M}-\mathrm{H}]^{-}, 100\right)$.

\section{ASSOCIATED CONTENT}

\section{S Supporting Information}

The Supporting Information is available free of charge on the ACS Publications website at DOI: 10.1021/acs.joc.7b01225.

${ }^{1} \mathrm{H}$ and ${ }^{13} \mathrm{C}$ NMR spectra for all compounds (PDF)

\section{AUTHOR INFORMATION}

\section{Corresponding Author}

*E-mail: andrew.sutherland@glasgow.ac.uk.

ORCID $\odot$

Andrew Sutherland: 0000-0001-7907-5766

Notes

The authors declare no competing financial interest.

\section{ACKNOWLEDGMENTS}

Financial support from the Ministry of Higher Education and Scientific Research, Omar Al-Mukhtar University, Libya (studentship to M.A.B.M.), EPSRC (EP/K503903/1), and the University of Glasgow is gratefully acknowledged.

\section{REFERENCES}

(1) For reviews, see: (a) Hassan, J.; Sévignon, M.; Gozzi, C.; Schulz, E.; Lemaire, M. Chem. Rev. 2002, 102, 1359. (b) Nicolaou, K. C.; Bulger, P. G.; Sarlah, D. Angew. Chem., Int. Ed. 2005, 44, 4442. (c) Cernak, T.; Dykstra, K. D.; Tyagarajan, S.; Vachal, P.; Krska, S. W. Chem. Soc. Rev. 2016, 45, 546.

(2) (a) Engvild, K. C. Phytochemistry 1986, 25, 781. (b) Gribble, G. W. Acc. Chem. Res. 1998, 31, 141. (c) Gribble, G. W. Chem. Soc. Rev. 1999, 28, 335. (d) Gribble, G. W. Chemosphere 2003, 52, 289.

(3) Lang, M.; Spiteller, P.; Hellwig, V.; Steglich, W. Angew. Chem., Int. Ed. 2001, 40, 1704.

(4) Ando, K.; Kato, A.; Suzuki, S. Biochem. Biophys. Res. Commun. 1970, 39, 1104.

(5) (a) Bohlmann, F.; Abraham, W.-R. Phytochemistry 1979, 18, 839. (b) Bohlmann, F.; Knauf, W.; Misra, L. N. Tetrahedron 1984, 40, 4987. (6) (a) Brownlee, G.; Copp, F. C.; Duffin, W. M.; Tonkin, I. M. Biochem. J. 1943, 37, 572. (b) Mitchell, A. G. J. Pharm. Pharmacol. 1964, 16, 533.

(7) (a) Peters, E. J.; Lowance, S. A. Agronomy J. 1970, 62, 400. (b) Peters, E. J.; Lowance, S. A. Weed Sci. 1972, 20, 140.

(8) (a) De La Mare, P. B. D. Electrophilic Halogenation; Cambridge University Press: New York, 1976. (b) Larock, R. C. Comprehensive Organic Transformations, 2nd ed.; Wiley-VCH: New York, 1999; pp 619-626.

(9) Hodgson, H. H. Chem. Rev. 1947, 40, 251.

(10) Snieckus, V. Chem. Rev. 1990, 90, 879.

(11) For some metal-free examples of aryl chlorination, see: (a) Yadav, J. S.; Reddy, B. V. S.; Reddy, P. S. R.; Basak, A. K.; Narsaiah, A. V. Adv. Synth. Catal. 2004, 346, 77. (b) Prakash, G. K. S.; Mathew, T.; Hoole, D.; Esteves, P. M.; Wang, Q.; Rasul, G.; Olah, G. A. J. Am. Chem. Soc. 2004, 126, 15770. (c) Maddox, S. M.; Dinh, A. N.; Armenta, F.; Um, J.; Gustafson, J. L. Org. Lett. 2016, 18, 5476.

(12) For reviews, see: (a) Sheppard, T. D. Org. Biomol. Chem. 2009, 7, 1043. (b) Petrone, D. A.; Ye, J.; Lautens, M. Chem. Rev. 2016, 116, 8003.

(13) For examples, see: (a) Tanemura, K.; Suzuki, T.; Nishida, Y.; Satsumabayashi, K.; Horaguchi, T. Chem. Lett. 2003, 32, 932. (b) Mo, F.; Yan, J. M.; Qiu, D.; Li, F.; Zhang, Y.; Wang, J. Angew. Chem., Int. Ed. 2010, 49, 2028. (c) Shen, X.; Hyde, A. M.; Buchwald, S. L. J. Am.
Chem. Soc. 2010, 132, 14076. (d) Xu, J.; Zhu, X.; Zhou, G.; Ying, B.; Ye, P.; Su, L.; Shen, C.; Zhang, P. Org. Biomol. Chem. 2016, 14, 3016. (14) For some recent examples, see: (a) Zhan, B.-B.; Liu, Y.-H.; Hu, F.; Shi, B.-F. Chem. Commun. 2016, 52, 4934. (b) Lied, F.; Lerchen, A.; Knecht, T.; Mück-Lichtenfeld, C.; Glorius, F. ACS Catal. 2016, 6, 7839. (c) Liu, X.-H.; Park, H.; Hu, J.-H.; Hu, Y.; Zhang, Q.-L.; Wang, B.-L.; Sun, B.; Yeung, K.-S.; Zhang, F.-L.; Yu, J.-Q. J. Am. Chem. Soc. 2017, 139, 888 and references cited therein.

(15) Shi, H.; Wang, P.; Suzuki, S.; Farmer, M. E.; Yu, J.-Q. J. Am. Chem. Soc. 2016, 138, 14876.

(16) (a) Racys, D. T.; Warrilow, C. E.; Pimlott, S. L.; Sutherland, A. Org. Lett. 2015, 17, 4782. (b) Racys, D. T.; Sharif, S. A. I.; Pimlott, S. L.; Sutherland, A. J. Org. Chem. 2016, 81, 772.

(17) Mostafa, M. A. B.; Calder, E. D. D.; Racys, D. T.; Sutherland, A. Chem. - Eur. J. 2017, 23, 1044.

(18) (a) Earle, M.; McAuley, B. J.; Ramani, A.; Seddon, K.; Thompson, J. WO 02072260, 2002. (b) Earle, M. J.; Hakala, U.; McAuley, B. J.; Nieuwenhuyzen, M.; Ramani, A.; Seddon, K. R. Chem. Commun. 2004, 1368.

(19) Antoniotti, S.; Dalla, V.; Duñach, E. Angew. Chem., Int. Ed. 2010, 49, 7860.

(20) As presented in Table 1 (entry 2), attempted chlorination of anisole in [BMIM] NTf $2\left(50{ }^{\circ} \mathrm{C}, 24 \mathrm{~h}\right)$ without the presence of iron(III) chloride results in no conversion. This in contrast to the results from ref $11 \mathrm{a}$, in which it is reported that chlorination of anisole with NCS using only the ionic liquid $[\mathrm{BMIM}] \mathrm{PF}_{6}$ at $27{ }^{\circ} \mathrm{C}$ gave an $87 \%$ yield after $65 \mathrm{~min}$. We repeated this reaction under the same conditions, and after $65 \mathrm{~min}$, we observed no conversion. After $24 \mathrm{~h}$, there was less than $5 \%$ conversion. We also repeated the reaction at 50 ${ }^{\circ} \mathrm{C}$ for $60 \mathrm{~h}$ and observed $5 \%$ conversion. These results confirm our observations that to perform chlorination reactions of electron-rich arenes, an activating species in addition to the presence of an ionic liquid is required. This is corroborated by other reports that have used Brønsted acid activated ionic liquids and elevated temperatures to achieve chlorination of electron-rich aromatic compounds. For example, see: (a) Hubbard, A.; Okazaki, T.; Laali, K. K. Aust. J. Chem. 2007, 60, 923. (b) Vražič, D.; Jereb, M.; Laali, K. K.; Stavber, S. Molecules 2013, 18, 74.

(21) Hjelm, O.; Borén, H.; Öberg, G. Chemosphere 1996, 32, 1719.

(22) Aziz-ur-Rehman; Rasool, S.; Abbasi, M. A.; Nafessa, K.; Fatima, A.; Gul, S.; Hussain, G.; Khan, K. M.; Ahmad, I.; Afzal, S. J. Chem. Soc. Pak. 2014, 36, 446.

(23) Aziz-ur-Rehman; Fatima, A.; Abbasi, M. A.; Khan, K. M.; Ashraf, M.; Ahmad, I.; Ejaz, S. A. Asian J. Chem. 2013, 25, 3735.

(24) Feng, X.; Qu, Y.; Han, Y.; Yu, X.; Bao, M.; Yamamoto, Y. Chem. Commun. 2012, 48, 9468.

(25) Hirano, M.; Yakabe, S.; Monobe, H.; Morimoto, T. Can. J. Chem. 1997, 75, 1905.

(26) Häußler, D.; Gütschow, M. Synthesis 2016, 48, 245.

(27) Liu, Y.; Park, S. K.; Xiao, Y.; Chae, J. Org. Biomol. Chem. 2014, $12,4747$.

(28) Minami, N.; Kijima, S. Chem. Pharm. Bull. 1979, 27, 816.

(29) Crocker, H. P.; Walser, R. J. Chem. Soc. C 1970, 1982.

(30) Noshita, M.; Shimizu, Y.; Morimoto, H.; Ohshima, T. Org. Lett. 2016, 18, 6062

(31) Wang, H.; Wen, K.; Nurahmat, N.; Shao, Y.; Zhang, H.; Wei, C.; Li, Y.; Shen, Y.; Sun, Z. Beilstein J. Org. Chem. 2012, 8, 744.

(32) van der Werf, A.; Selander, N. Org. Lett. 2015, 17, 6210.

(33) Mąkosza, M.; Białecki, M. J. Org. Chem. 1998, 63, 4878.

(34) Stoll, A. H.; Knochel, P. Org. Lett. 2008, 10, 113.

(35) Sonawane, R. B.; Rasal, N. K.; Jagtap, S. V. Org. Lett. 2017, 19, 2078.

(36) Pu, X.; Li, Q.; Lu, Z.; Yang, X. Eur. J. Org. Chem. 2016, 5937.

(37) Thorat, P. B.; Bhong, B. Y.; Karade, N. N. Synlett 2013, 24, 2061.

(38) Liu, J.-H.; Yang, C.-T.; Lu, X.-Y.; Zhang, Z.-Q.; Xu, L.; Cui, M.; Lu, X.; Xiao, B.; Fu, Y.; Liu, L. Chem. - Eur. J. 2014, 20, 15334.

(39) Xu, J.; Wang, X.; Shao, C.; Su, D.; Cheng, G.; Hu, Y. Org. Lett. 2010, 12, 1964. 
(40) Wiley, R. H.; Wakefield, B. J. J. Org. Chem. 1960, 25, 546.

(41) Ngi, S. I.; Petrignet, J.; Duwald, R.; Hilali, E. M. E.; Abarbri, M.; Duchêne, A.; Thibonnet, J. Adv. Synth. Catal. 2013, 355, 2936.

(42) Wyrick, S. D.; Smith, F. T.; Kemp, W. E.; Grippo, A. A. J. Med. Chem. 1987, 30, 1798.

(43) Yin, W.-P.; Shi, M. Tetrahedron 2005, 61, 10861.

(44) Slocum, D. W.; Reece, T. L.; Sandlin, R. D.; Reinscheld, T. K.; Whitley, P. E. Tetrahedron Lett. 2009, 50, 1593.

(45) Scheepstra, M.; Nieto, L.; Hirsch, A. K. H.; Fuchs, S.; Leysen, S.; Lam, C. V.; in het Panhuis, L.; van Boeckel, C. A. A.; Wienk, H.; Boelens, R.; Ottmann, C.; Milroy, L.-G.; Brunsveld, L. Angew. Chem., Int. Ed. 2014, 53, 6443. 